\title{
Hidden star-formation in the cluster of galaxies Abell 1689^
}

\author{
P.-A. Duc ${ }^{1,2}$, B. M. Poggianti ${ }^{3}$, D. Fadda ${ }^{4,2}$, D. $\operatorname{Elbaz}^{2,5}$, H. Flores ${ }^{6,2}$, P. Chanial ${ }^{2}$, A. Franceschini ${ }^{7}$, \\ A. Moorwood $^{8}$, and C. Cesarsky ${ }^{8}$
}

\section{${ }^{1}$ CNRS URA 2052}

2 CEA, DSM, DAPNIA, Service d'astrophysique, 91191 Gif-sur-Yvette Cedex, France

3 Osservatorio Astronomico di Padova, vicolo dell'Osservatorio 5, 35122 Padova, Italy

4 Instituto de Astrofisica de Canarias, Via Lactea s/n, 38200 La Laguna - Tenerife, Spain

5 Department of Astronomy \& Astrophysics, University of California, Santa Cruz, CA 95064,USA

6 DAEC/LUL, Observatoire de Paris-Meudon, 5 place Jules Janssen, 92195 Meudon, France

7 Dipartimento di Astronomia, Università di Padova, Vicolo dell'Osservatorio, 5, 35122 Padova, Italy

8 European Southern Observatory, Karl-Schwarzchild-Strasse, 285748 Garching bei München, Germany

Received 5 October 2001 / Accepted 28 November 2001

\begin{abstract}
At a redshift of 0.18 , Abell 1689 is so far the most distant cluster of galaxies for which substantial mid-infrared (MIR) data have been published. Its mapping with the ISOCAM camera onboard the ISO satellite allowed the detection of 30 cluster members at $6.75 \mu \mathrm{m}$ ( $L W 2$ filter) and 16 cluster members at $15 \mu \mathrm{m}$ ( $L W 3$ filter) within a clustercentric radius of $0.5 \mathrm{Mpc}$ (Fadda et al. 2000a, Paper I). We present here the follow-up optical photometric and spectroscopic observations which were used to study the individual properties of the galaxy members of A1689. We confirm the high fraction of blue galaxies initially reported in this rich cluster by Butcher \& Oemler (1984), that was challenged by some subsequent studies. We discuss the spectral and morphological properties of all cluster members in our spectroscopic sample, and of the MIR-detected galaxies in particular. Sources with a low $[15 \mu \mathrm{m}] /[6.75 \mu \mathrm{m}]$ flux ratio typically consist of luminous passive early-type galaxies while those with a high MIR color index are mainly luminous, blue, emission-line, morphologically disturbed spirals, i.e. the star-forming galaxies usually associated with the "Butcher-Oemler" effect. On the other hand, at least $30 \%$ of the $15 \mu \mathrm{m}$ sources have optical counterparts showing no evidence for current star-formation activity, while their $15 \mu \mathrm{m}$ emission is most likely due to obscured star formation. We argue that the $L W 3$ luminosity measured in the cluster members is a reliable tracer of the total infrared luminosity which in A1689 galaxies peaks at $L_{\mathrm{IR}}=6.2 \times 10^{10} L_{\odot}$. We derive from $L_{\mathrm{IR}}$ a star-formation rate free of dust extinction, $S F R(\mathrm{IR})$, which we compare with that determined in the optical from the flux of the [OII] emission line, $S F R$ (opt). The highest total star formation rates $\left(11 M_{\odot} \mathrm{yr}^{-1}\right)$ and dust extinction are measured in those galaxies exhibiting in their optical spectrum a signature of a dusty starburst. In contrast, none of the galaxies with post-starburst optical spectra has been detected by ISOCAM down to a $15 \mu \mathrm{m}$ flux limit corresponding to $1.4 M_{\odot} \mathrm{yr}^{-1}$. We find a median $S F R(\mathrm{IR})$ of the $L W 3$-detected galaxies of $2 M_{\odot} \mathrm{yr}^{-1}$ that is ten times higher than the median $S F R(\mathrm{opt})$ of the [OII]- detected galaxies. The ratio $S F R(\mathrm{IR}) / S F R(\mathrm{opt})$ is in fact very high, ranging between 10 and 100 for $L W 3$-detected galaxies with [OII] emission. We conclude that a major part, at least $90 \%$, of the star formation activity taking place in Abell 1689 is hidden. Whether the high extinction measured in the star-forming cluster members results from the cluster environment itself or reflects a comparable extinction in the coeval field is still unclear.
\end{abstract}

Key words. galaxies: clusters: individual: Abell 1689 - galaxies: evolution - infrared: galaxies

\section{Introduction}

The history of star formation (SF) as a function of redshift has recently received extensive attention since the reference work by Madau et al. (1996). Combining optical observations on field galaxies in the nearby $(z=0)$, intermediate redshift $(z<1)$ and distant $(1<z<4)$

\footnotetext{
Send offprint requests to: P.-A. Duc, e-mail: paduc@cea.fr

* Based on observations collected at the European Southern Observatory, La Silla, Chile (ESO No. 61.A-0619).
}

universe, they highlighted the strong rise of the star formation comoving density up to $z=1$ and its possible decline at higher redshift. Further multiwavelength studies have added points on the curve and addressed various biases and uncertainties in the slopes of the SF rise and decline. In contrast, the global star formation history in clusters is practically unknown (See though Kodama \& Bower 2001). A number of impediments make its study particularly difficult. First of all, data for distant clusters $(z>1)$ are still missing. Furthermore, variations in 
cluster properties, such as the X-ray luminosity, the level of sub-clustering and, generally, the dynamical status of the cluster, introduce a large dispersion in any trend. A "global" history - even just limited to rich clusters - can only be obtained using large samples. Also, the star formation history of clusters is largely related to the star formation history in the coeval field via the infall rate of field galaxies which is itself redshift-dependent as well. Any uncertainty in the latter has a strong impact on the former. Finally, problems with dust obscuration are critical when computing the field star formation rate $(S F R)$ density, and they could be at least as annoying in clusters.

The long-term effect of the cluster environment is undoubtedly to quench star-formation in its member galaxies (e.g. Abraham et al. 1996; Poggianti et al. 1999; Balogh et al. 1999). Indeed, nearby rich clusters contain more passive, non-starforming galaxies than more distant ones. This is one aspect of the so-called Butcher-Oemler effect which is the observed increase in the number of blue, presumably star-forming galaxies in clusters, as a function of redshift (Butcher \& Oemler 1984, hereafter BO84). Furthermore, at intermediate redshifts the star formation rate per cluster galaxy appears to be lower than in similar types of galaxies in the surrounding field (e.g. Dressler et al. 1999; Balogh et al. 2000). Unfortunately, estimates of the $S F R$ rely on measurements of optical line fluxes, often [OII], obtained with aperture limited slits. Some H II regions might have been missed and hence the $S F R$ underestimated. Data from narrow-band $\mathrm{H}_{\alpha}$ images have recently become available for a few $z=0.2-0.3$ clusters (Balogh \& Morris 2000). This kind of data has the advantage of covering the whole galaxy, but it is less sensitive to low levels of star formation activity. Moreover, the $S F R$ derived in the optical might suffer from strong dust extinction. A constant dust absorption of $1 \mathrm{mag}$ at $\mathrm{H}_{\alpha}$, typical of local spirals (Kennicutt 1992), is usually assumed to correct the optically-derived $S F R$. However, dust obscuration varies dramatically from one galaxy to another, and it is likely to depend also on the environmental conditions, as will be discussed in this paper. Direct optical signatures of dust enshrouded starbursts have already been found in some cluster galaxies that have a spectrum classified as, according to the authors, "e(a)" (Dressler et al. 1999), "a+em" (Balogh \& Morris 2000) or "S+A" (in the field, Flores et al. 1999). All these spectra show a moderate $[\mathrm{OII}]_{\lambda 3727}$ line in emission and strong early Balmer absorption lines. Such features are well reproduced by spectrophotometric models with a selective dust extinction which affects differently young and older stars (Poggianti et al. 2001). Though this spectral combination is able to reveal the occurrence of some level of hidden star formation, the models have shown that it is impossible to quantify the total $S F R$ on the basis of optical observations alone.

Furthermore, several spectroscopic surveys of distant clusters (e.g. Couch \& Sharples 1987; Fabricant et al. 1991; Barger et al. 1998; Fisher et al. 1998; Dressler et al. 1999) have disclosed a significant population of $\mathrm{k}+\mathrm{a}$ galaxies whose spectrum is characterized by the absence of emission lines and the presence of strong Balmer lines in absorption. Classically, such objects are considered as post-starburst or post-starforming galaxies (e.g. Couch \& Sharples 1987; Poggianti et al. 1999). Could they however be extreme e(a) galaxies in which star formation is totally obscured at optical wavelengths, as suggested by Smail et al. (1999) from radio-continuum observations? In addition, the post-starburst interpretation of the strongest $\mathrm{k}+\mathrm{a}$ 's requires that a starburst episode occurred in these galaxies, possibly when they fell in the cluster. Optical surveys have yet failed to pinpoint the putative starbursting progenitors, perhaps for time scale reasons (Couch et al. 2001), and "e(a)" galaxies have been suggested to be the most likely progenitors of the post-starburst galaxies (Poggianti et al. 1999).

Undoubtedly deep mid-infrared surveys can address some of the previous questions. Fluxes at 10-15 $\mu \mathrm{m}$, along with radio centimetric fluxes, provide the best estimates of the dust obscured star formation rates (e.g. Elbaz et al. 2001) when far-infrared data are not available. The latest generation of infrared cameras, such as ISOCAM (Cesarsky et al. 1996) on board the ISO, have provided for the first time images in the mid-infrared regime with a field of view, spatial resolution and sensitivity (Genzel \& Cesarsky 2000) particularly well suited to the study of distant clusters.

We made use of the unique capabilities of ISOCAM to map at $6.75 \mu \mathrm{m}$ ( $L W 2$ filter, bandwidth: $5.0-8.3 \mu \mathrm{m}$ ) and $15 \mu \mathrm{m}$ ( $L W 3$ filter, bandwidth: 11.6-18.0 $\mu \mathrm{m}$ ) a sample of ten clusters in the redshift range $0.2-0.9$ (Fadda et al. 2000a). The mid-infrared data of the most nearby cluster of our sample, Abell 1689, were presented in Fadda et al. (2000b, hereafter Paper I). Towards this rich cluster, situated at a redshift of $0.18^{1}$, we detected 45 infrared sources (41 LW2 sources and $25 L W 3$ sources). Among them, $6(13 \%)$ sources were identified as stars and $8(18 \%)$ turn out to be foreground or background objects. A1689 was found to contain a population of galaxies with high $15 \mu \mathrm{m}$ fluxes and high MIR to optical flux ratios, while such population is absent towards the central region of the Virgo and Coma clusters. This was interpreted as revealing an "infrared Butcher-Oemler effect". Moreover, the comparison of optical and MIR counts in the field and towards A1689 seemed to suggest a possible excess in the number of MIR emitters in the cluster as compared to the number expected given the different spiral fraction in the two environments and the fact that most of the $L W 3$ emitters are spiral galaxies. Such an excess would indicate that SF is triggered in some galaxies by the cluster environment. The $15 \mu \mathrm{m}$ luminosities and MIR-to-optical flux ratios,

\footnotetext{
${ }^{1}$ We have taken $H_{0}=75 \mathrm{~km} \mathrm{~s}^{-1} \mathrm{Mpc}^{-1}$ and $q_{0}=0.5$. Adopting this cosmology, the distance of Abell 1689 is $767 \mathrm{Mpc}$, which is the value used throughout the paper. With a more typical cosmology, such as $\Omega_{m}=0.3, \Omega_{\lambda}=0.7$, and $H_{0}=70 \mathrm{~km} \mathrm{~s}^{-1} \mathrm{Mpc}^{-1}$, the distance would become $877 \mathrm{Mpc}$. At the adopted distance of Abell 1689, 1 arcmin corresponds to $159 \mathrm{kpc}$.
} 
instead, were not found to be significantly different in the cluster and in the coeval field.

In the present paper, we focus on the properties of the galaxies members of A1689 that were detected with ISOCAM, comparing the optical and the IR view of the Butcher-Oemler effect. A major goal of this work is to establish the amount of SF hidden by dust in the galaxies of A1689, and whether a population of starburst galaxies is present in the cluster but would be unrecognized on the basis of the optical data.

Our study relies on an extensive spectroscopic survey of the cluster that has been carried out with the ESO New Technology Telescope (NTT) and on a collection of new and previous photometric data. The literature on Abell 1689 is indeed very rich. The cluster was included in the original paper highlighting what was later known as the Butcher-Oemler effect (BO84). Several authors have obtained additional photometric data and discussed whether the rather large blue fraction found in A1689 by BO84 was real or not (e.g. Gudehus \& Hegyi 1991; Pickles \& van der Kruit 1991; Molinari et al. 1996; Margoniner \& de Carvalho 2000). The lensing properties of the cluster, studied through deep optical images, were used to determine its mass profile (Tyson \& Fischer 1995; Dye et al. 2001). The latter was also estimated from X-ray maps (Miralda-Escude \& Babul 1995). Evidence for dynamical substructures was found by Teague et al. (1990) based on a set of spectroscopic data.

The paper is organized as follows: in Sect. 2 we present our spectroscopic and photometric observations and compare them with data from the literature. In Sect. 3, we describe the properties of the optically-selected (Sect. 3.2) and MIR-selected cluster members (Sect. 3.3). Finally, in Sect. 4, we discuss the star formation activity and the dust extinction of the galaxies in the cluster and provide some clues on environmental effects that affected their evolution.

\section{Observations and data-reduction}

\subsection{MIR data}

The observations and data reduction of the ISOCAM midinfrared data have been described in detail in Paper I. We recall here the basic characteristics of the survey. The total field of view was $6^{\prime} \times 6^{\prime}$ (corresponding to $0.95 \times 0.95 \mathrm{Mpc}$ ) with a pixel size of $6^{\prime \prime}$. The spatial resolution and astrometry were good enough to allow an unambiguous optical identification of all the mid-infrared sources but the ones near the very crowded cluster center. The $90 \%$ completeness limits were 0.2 and $0.4 \mathrm{mJy}$ at $6.75 \mu \mathrm{m}$ and $15 \mu \mathrm{m}$, respectively. The sensitivity limits of the $L W 2$ and $L W 3$ surveys were $0.15 \mathrm{mJy}$ and $0.3 \mathrm{mJy}$, respectively.

\subsection{Imaging data}

$B^{2}, V$ and $R$ images of the cluster have been obtained during the spectroscopic run which was carried out in May 1998 at the New-Technology Telescope at La Silla observatory. The seeing was poor (about 1.5"). Landolt fields of photometric standard stars (Landolt 1992) were observed for flux calibration. Due to the atmospheric conditions, the accuracy of the absolute photometric zeropoints is no better than 0.05 mag. The astrometry of each frame was computed using several tens of reference stars from the USNO A1.0 catalog (Monet 1996) queried via the ESO SKYCAT browser. The images were corrected for distortions during this process. Sources were extracted from the reduced $B V R$ images using SExtractor (Bertin $\&$ Arnouts 1996). The photometric catalog is complete to $B=23 \mathrm{mag}, V=22.7 \mathrm{mag}$ and $R=22.7 \mathrm{mag}$. The corrected isophotal ("mag_best") $B V R$ magnitudes from SExtractor are listed in Tables 1-4.

Our photometric database also includes the Gunn $g, r$ and $i$ magnitudes measured by Molinari et al. (1996) with the ESO $3.6 \mathrm{~m}$ telescope and independently by Margoniner \& de Carvalho (2000) with the CTIO $0.9 \mathrm{~m}$ telescope, the $B$ and $I$ magnitudes measured by Dye et al. (2001) at the Calar-Alto $3.5 \mathrm{~m}$ telescope and the nearinfrared $K^{\prime}$-band magnitudes measured by de Propris et al. (1999) at the CTIO $1.5 \mathrm{~m}$. All these different catalogs were cross-identified. Unfortunately because these data have various origins and were extracted with different methods, they cannot be easily compared. In particular they turn out to be of little use to derive a reliable spectral energy distribution. In the following, we will thus mostly rely on our own $B V R$ photometric data.

Finally, archive HST/WCPC2 images (PI: Tyson) covering the whole MOS field of view have been processed by the MORPHS group and were kindly given to us. They consist of recombined $F 555 W$ and $F 814 W$ images. The HST astrometry was lost during the combination process and it was re-computed using the astrometric solution of our ground-base images as reference.

\subsection{Spectroscopic data}

The spectroscopic observations were carried out with EMMI installed on the NTT. Using five masks punched before the observations, we obtained spectra of 111 different objects towards the inner $\mathrm{Mpc}^{2}\left(5^{\prime} \times 7^{\prime}\right)$ of Abell 1689. Each slitlet was $1.3^{\prime \prime}$ wide $(3.4 \mathrm{kpc}$ at the distance of A1689) and at least $8^{\prime \prime}$ long. The slit length was actually adjusted to cover the whole length of the target object keeping some blank sky at each side. Note that, due to geometrical constraints, the slit orientation was not necessarily along the main axis. The targets were selected from a deep $V$-band image of the cluster which was available in the NTT archive. We chose preferentially

\footnotetext{
2 The EMMI red channel blue-like Bb filter which was used has a transmission curve slightly different from the standard Johnson's filter.
} 
Table 1. Spectroscopically-confirmed cluster members in the MOS sample.

\begin{tabular}{|c|c|c|c|c|c|c|c|c|}
\hline $\begin{array}{l}\text { ID } \\
(\mathrm{MOS})\end{array}$ & $\begin{array}{c}\text { ID }^{*} \\
\text { (ISO) }\end{array}$ & $\begin{array}{c}\mathrm{RA} \\
\mathrm{J} 2000\end{array}$ & $\begin{array}{c}\text { DEC } \\
\text { J2000 }\end{array}$ & $\begin{array}{c}z \\
(\operatorname{MOS})\end{array}$ & $\begin{array}{c}B \\
\text { mag }\end{array}$ & $\begin{array}{c}V \\
\text { mag }\end{array}$ & $\begin{array}{c}R \\
\text { mag }\end{array}$ & morph \\
\hline 1 & - & $13: 11: 24.00$ & $-1: 19: 07.6$ & 0.1829 & 21.53 & 19.98 & 19.24 & - \\
\hline 2 & - & $13: 11: 24.25$ & $-1: 21: 14.8$ & 0.1766 & 21.41 & 20.82 & 20.27 & Scd \\
\hline 3 & - & $13: 11: 24.27$ & $-1: 18: 38.1$ & 0.1803 & 21.87 & 20.38 & 19.65 & $\mathrm{Sb}$ \\
\hline 4 & - & $13: 11: 24.40$ & $-1: 21: 11.5$ & 0.1870 & 20.72 & 19.11 & 18.42 & so \\
\hline 5 & - & $13: 11: 24.41$ & $-1: 22: 15.8$ & 0.1785 & 20.48 & 18.81 & 18.08 & - \\
\hline 6 & - & $13: 11: 25.07$ & $-1: 19: 31.7$ & 0.1797 & 21.44 & 19.79 & 19.07 & So \\
\hline 7 & 3 & $13: 11: 25.31$ & $-1: 20: 37.6$ & 0.1924 & 19.75 & 18.13 & 17.38 & So \\
\hline 8 & .. & $13: 11: 25.64$ & $-1: 17: 24.8$ & 0.1814 & 20.91 & 19.31 & 18.59 & $\mathrm{E}$ \\
\hline 9 & - & $13: 11: 25.94$ & $-1: 19: 36.0$ & 0.1983 & 21.81 & 20.48 & 19.77 & Sab \\
\hline 10 & - & $13: 11: 26.51$ & $-1: 22: 01.4$ & 0.1953 & 21.21 & 19.66 & 18.98 & $\mathrm{Sc}$ \\
\hline 11 & .. & $13: 11: 27.03$ & $-1: 16: 10.5$ & 0.1945 & 20.08 & 18.86 & 18.13 & $\mathrm{Sbc}$ \\
\hline 12 & - & $13: 11: 27.05$ & $-1: 18: 49.2$ & 0.1852 & 22.36 & 20.88 & 20.09 & $\mathrm{Sa}$ \\
\hline 13 & - & $13: 11: 27.06$ & $-1: 21: 43.4$ & 0.1955 & 22.52 & 21.28 & 20.68 & So \\
\hline 14 & 4 & $13: 11: 27.07$ & $-1: 20: 58.9$ & 0.2153 & 20.81 & 19.68 & 19.10 & Scd \\
\hline 15 & - & $13: 11: 27.37$ & $-1: 22: 48.2$ & 0.1832 & 21.13 & 19.43 & 18.73 & - \\
\hline 16 & 6 & $13: 11: 27.69$ & $-1: 21: 06.7$ & 0.2165 & 20.59 & 19.50 & 19.06 & $\mathrm{Sd}$ \\
\hline 17 & - & $13: 11: 27.80$ & $-1: 21: 13.4$ & 0.1977 & 21.55 & 19.95 & 19.19 & - \\
\hline 18 & - & $13: 11: 27.86$ & $-1: 21: 37.2$ & 0.1752 & 21.13 & 19.66 & 18.94 & So \\
\hline 19 & - & $13: 11: 27.98$ & $-1: 18: 44.5$ & 0.1890 & 21.26 & 19.67 & 18.89 & So \\
\hline 20 & - & $13: 11: 28.15$ & $-1: 18: 12.7$ & 0.1902 & 22.16 & 20.44 & 19.85 & So \\
\hline 21 & 8 & $13: 11: 28.16$ & $-1: 20: 44.0$ & 0.1712 & 20.34 & 18.75 & 18.04 & So \\
\hline 22 & - & $13: 11: 28.29$ & $-1: 18: 45.5$ & 0.1999 & 21.04 & 19.60 & 18.92 & - \\
\hline 23 & 10 & $13: 11: 28.30$ & $-1: 19: 58.9$ & 0.1780 & 20.29 & 18.95 & 18.34 & $\mathrm{Sab}$ \\
\hline 24 & - & $13: 11: 28.42$ & $-1: 22: 42.1$ & 0.1842 & 20.48 & 18.82 & 17.96 & - \\
\hline 25 & - & $13: 11: 28.56$ & $-1: 20: 27.1$ & 0.1909 & 21.34 & 19.77 & 19.08 & So \\
\hline 26 & - & $13: 11: 28.69$ & $-1: 19: 03.3$ & 0.1859 & 21.91 & 20.21 & 19.44 & - \\
\hline 27 & - & $13: 11: 29.26$ & $-1: 19: 17.3$ & 0.1932 & 21.30 & 19.57 & 18.89 & So \\
\hline 28 & 12 & $13: 11: 29.27$ & $-1: 17: 50.4$ & 0.1726 & 20.34 & 19.24 & 18.79 & $\mathrm{Sab}$ \\
\hline 29 & 14 & $13: 11: 29.44$ & $-1: 20: 28.4$ & 0.1842 & 18.42 & 16.87 & 16.02 & Scd \\
\hline 30 & .. & $13: 11: 29.71$ & $-1: 17: 22.1$ & 0.1996 & 20.87 & 19.20 & 18.43 & so \\
\hline 31 & - & $13: 11: 29.74$ & $-1: 17: 43.2$ & 0.1830 & 22.45 & 21.11 & 20.44 & $\mathrm{Sbc}$ \\
\hline 32 & - & $13: 11: 29.83$ & $-1: 20: 15.8$ & 0.2016 & 21.82 & 20.11 & 19.27 & $\mathrm{Sb}$ \\
\hline 33 & 16 & $13: 11: 29.94$ & $-1: 20: 40.8$ & 0.2002 & 19.83 & 18.72 & 18.08 & $\mathrm{Sp}$ \\
\hline 34 & - & $13: 11: 29.95$ & $-1: 22: 07.9$ & 0.1985 & 20.48 & 18.96 & 18.21 & So \\
\hline 35 & - & $13: 11: 29.96$ & $-1: 20: 17.9$ & 0.1801 & 21.62 & 19.96 & 19.28 & $\mathrm{~S} 0 / \mathrm{a}$ \\
\hline 36 & .. & $13: 11: 29.98$ & $-1: 16: 25.6$ & 0.1785 & 21.17 & 19.56 & 18.84 & - \\
\hline 37 & 18 & $13: 11: 30.01$ & $-1: 20: 43.3$ & 0.2038 & 19.53 & 18.10 & 17.34 & - \\
\hline 38 & - & $13: 11: 30.11$ & $-1: 22: 30.8$ & 0.1910 & 20.81 & 19.34 & 18.67 & So \\
\hline 39 & 20 & $13: 11: 30.23$ & $-1: 20: 29.8$ & 0.1745 & 19.53 & 17.87 & 17.17 & $\mathrm{E}$ \\
\hline 40 & 22 & $13: 11: 30.53$ & $-1: 20: 44.3$ & 0.1918 & 20.40 & 18.78 & 17.91 & $\mathrm{E}$ \\
\hline 41 & - & $13: 11: 31.03$ & $-1: 21: 28.3$ & 0.1878 & 20.28 & 18.50 & 17.87 & $\mathrm{E}$ \\
\hline 42 & 24 & 13:11:31.09 & $-1: 21: 25.7$ & 0.1972 & 20.93 & 19.42 & 18.32 & $\mathrm{E}$ \\
\hline 43 & 26 & $13: 11: 31.36$ & $-1: 19: 33.4$ & 0.1878 & 19.54 & 17.81 & 17.03 & $\mathrm{E}$ \\
\hline 44 & - & $13: 11: 31.47$ & $-1: 19: 25.5$ & 0.1745 & 21.27 & 19.74 & 19.05 & $\mathrm{Sbc}$ \\
\hline 45 & .. & $13: 11: 31.54$ & $-1: 17: 28.4$ & 0.1913 & 20.93 & 19.26 & 18.55 & so \\
\hline 46 & 30 & $13: 11: 32.07$ & $-1: 19: 47.5$ & 0.1801 & 20.37 & 18.43 & 17.72 & $\mathrm{Sa}$ \\
\hline 47 & - & $13: 11: 32.17$ & $-1: 22: 11.3$ & 0.1855 & 20.57 & 18.90 & 17.93 & - \\
\hline 48 & - & $13: 11: 32.43$ & $-1: 22: 18.6$ & 0.1794 & 21.35 & 20.46 & 19.98 & $?$ \\
\hline 49 & 31 & $13: 11: 32.60$ & $-1: 18: 42.1$ & 0.1757 & 19.44 & 18.17 & 17.65 & Scd \\
\hline 50 & - & $13: 11: 32.61$ & $-1: 18: 37.9$ & 0.1823 & - & 23.96 & 20.84 & So \\
\hline 51 & 32 & $13: 11: 32.62$ & $-1: 19: 59.3$ & 0.2022 & 19.13 & 17.39 & 16.63 & - \\
\hline 52 & 33 & $13: 11: 32.67$ & $-1: 19: 32.4$ & 0.2009 & 19.55 & 17.66 & 16.83 & So \\
\hline 53 &.. & $13: 11: 33.08$ & $-1: 17: 02.4$ & 0.1891 & 19.94 & 18.29 & 17.58 & - \\
\hline 54 & 35 & $13: 11: 34.02$ & $-1: 21: 02.5$ & 0.1813 & 19.73 & 18.49 & 17.94 & $\mathrm{Sa}$ \\
\hline 55 & 37 & $13: 11: 34.47$ & $-1: 18: 11.7$ & 0.1989 & 19.66 & 18.34 & 17.73 & $\mathrm{Sc}$ \\
\hline 56 & .. & $13: 11: 34.65$ & $-1: 17: 43.7$ & 0.1926 & 20.22 & 18.54 & 17.77 & $\mathrm{E}$ \\
\hline 57 & - & $13: 11: 34.72$ & $-1: 20: 59.8$ & 0.1895 & 20.90 & 19.19 & 18.49 & $\mathrm{E}$ \\
\hline 58 & .. & $13: 11: 35.10$ & $-1: 23: 18.3$ & 0.1925 & 21.42 & 19.90 & 19.37 & - \\
\hline 59 & 40 & $13: 11: 35.31$ & $-1: 21: 33.8$ & 0.1870 & 20.39 & 18.69 & 17.95 & - \\
\hline 60 & .. & $13: 11: 35.47$ & $-1: 17: 42.9$ & 0.1758 & 20.99 & 19.29 & 18.58 & $\mathrm{Sa}$ \\
\hline 61 & 41 & $13: 11: 35.55$ & $-1: 20: 13.0$ & 0.2000 & 19.86 & 18.76 & 18.26 & $\mathrm{Sc}$ \\
\hline 62 & - & $13: 11: 36.52$ & $-1: 18: 47.3$ & 0.1769 & 22.18 & 20.56 & 19.85 & $\mathrm{E}$ \\
\hline 63 & .. & $13: 11: 36.58$ & $-1: 22: 54.6$ & 0.1758 & 20.15 & 19.08 & 18.45 & - \\
\hline 64 & - & $13: 11: 37.01$ & $-1: 22: 32.4$ & 0.1878 & 21.73 & 20.31 & 19.59 & - \\
\hline 65 &.. & $13: 11: 37.12$ & $-1: 17: 07.7$ & 0.1851 & 20.88 & 19.21 & 18.42 & So \\
\hline 66 & - & $13: 11: 37.80$ & $-1: 19: 21.2$ & 0.1839 & 19.92 & 18.32 & 17.61 & $\mathrm{E}$ \\
\hline 67 &.. & $13: 11: 37.88$ & $-1: 22: 38.0$ & 0.1887 & 21.19 & 19.69 & 18.94 & - \\
\hline 68 &.. & $13: 11: 37.91$ & $-1: 18: 09.0$ & 0.1804 & 19.91 & 18.17 & 17.45 & $\mathrm{E}$ \\
\hline 69 & 43 & $13: 11: 38.23$ & $-1: 21: 05.7$ & 0.1964 & 20.17 & 18.82 & 18.21 & Scd \\
\hline 70 &.. & $13: 11: 39.31$ & $-1: 16: 49.8$ & 0.1845 & 20.13 & 18.48 & 17.86 & $\mathrm{E}$ \\
\hline 71 & .. & $13: 11: 39.56$ & $-1: 17: 50.1$ & 0.1977 & 20.86 & 19.28 & 18.51 & So \\
\hline 72 & 45 & 13:11:40.09 & $-1: 19: 52.3$ & 0.1880 & 19.87 & 19.13 & 18.70 & Sp(late) \\
\hline 73 &.. & $13: 11: 40.27$ & $-1: 18: 01.1$ & 0.1807 & 20.46 & 18.90 & 18.16 & - \\
\hline 74 & .. & $13: 11: 43.38$ & $-1: 19: 20.4$ & 0.1837 & 19.83 & 18.01 & 17.29 & - \\
\hline
\end{tabular}

* Galaxies detected at $15 \mu \mathrm{m}$ are highlighted. Those outside the ISOCAM field of view are indicated with "..". Undetected ones in both $L W 2$ and $L W 3$ filters are indicated with "-". 
Table 2. Spectroscopically-confirmed cluster members from Teague et al. (1990).

\begin{tabular}{|c|c|c|c|c|c|c|c|}
\hline $\begin{array}{l}\mathrm{RA} \\
\mathrm{J} 2000\end{array}$ & $\begin{array}{c}\text { DEC } \\
\text { J2000 }\end{array}$ & $\begin{array}{c}\text { ID } \\
\text { (ISO) }\end{array}$ & $z$ & $\begin{array}{c}B \\
\mathrm{mag}\end{array}$ & $\begin{array}{c}V \\
\operatorname{mag}\end{array}$ & $\begin{array}{c}R \\
\mathrm{mag}\end{array}$ & morph \\
\hline 13:11:18.37 & $-1: 18: 40.3$ & - & 0.2095 & 18.92 & 17.69 & 17.05 & S0 \\
\hline 13:11:20.74 & $-1: 20: 02.9$ & - & 0.1835 & 20.47 & 18.83 & 18.07 & $\mathrm{Sa}$ \\
\hline $13: 11: 21.53$ & $-1: 19: 44.2$ & - & 0.2171 & 20.76 & 19.13 & 18.41 & - \\
\hline 13:11:26.85 & $-1: 19: 37.5$ & - & 0.1754 & 20.34 & 19.02 & 18.38 & $\mathrm{E}$ \\
\hline 13:11:27.08 & $-1: 22: 09.5$ & - & 0.1840 & 21.17 & 19.41 & 18.63 & SB0 \\
\hline 13:11:27.89 & $-1: 23: 09.1$ & - & 0.1842 & 20.48 & 18.77 & 18.02 & - \\
\hline 13:11:28.96 & $-1: 21: 17.3$ & - & 0.1947 & 20.53 & 18.86 & 18.16 & $\mathrm{E}$ \\
\hline 13:11:29.04 & $-1: 21: 37.7$ & - & 0.1858 & 21.21 & 19.57 & 18.81 & S0 \\
\hline 13:11:29.08 & $-1: 21: 55.8$ & - & 0.1908 & 21.02 & 19.35 & 18.57 & S0 \\
\hline 13:11:29.35 & $-1: 18: 35.4$ & - & 0.1751 & 20.81 & 19.16 & 18.44 & - \\
\hline 13:11:30.00 & $-1: 20: 43.0$ & - & 0.1987 & - & - & - & - \\
\hline 13:11:30.20 & $-1: 20: 28.0$ & - & 0.1750 & - & - & - & - \\
\hline 13:11:30.50 & $-1: 20: 46.0$ & - & 0.1987 & - & - & - & - \\
\hline 13:11:30.90 & $-1: 20: 31.0$ & - & 0.1739 & - & - & - & - \\
\hline 13:11:31.00 & $-1: 21: 28.0$ & - & 0.1865 & - & - & - & - \\
\hline 13:11:31.04 & $-1: 20: 53.1$ & - & 0.1885 & 21.32 & 19.55 & 18.80 & $\mathrm{E}$ \\
\hline 13:11:31.30 & $-1: 19: 33.0$ & - & 0.1864 & - & - & - & - \\
\hline 13:11:32.06 & $-1: 21: 38.7$ & 29 & 0.1770 & 20.57 & 18.98 & 18.33 & So \\
\hline $13: 11: 32.57$ & $-1: 23: 52.1$ & - & 0.1865 & 20.30 & 18.62 & 17.87 & - \\
\hline 13:11:33.74 & $-1: 18: 44.7$ & - & 0.1849 & 20.79 & 19.08 & 18.37 & $\mathrm{E}$ \\
\hline 13:11:35.34 & $-1: 20: 43.4$ & - & 0.1835 & 21.21 & 19.54 & 18.80 & $\mathrm{E}$ \\
\hline 13:11:35.98 & $-1: 23: 41.4$ & - & 0.1861 & 20.29 & 18.54 & 17.76 & - \\
\hline 13:11:39.43 & $-1: 19: 07.2$ & - & 0.1813 & 20.39 & 19.02 & 18.40 & $\mathrm{Sa}$ \\
\hline
\end{tabular}

Table 3. ISOCAM cluster members with photometric redshift.

\begin{tabular}{cccccccc}
\hline $\begin{array}{c}\text { ID } \\
\text { (ISO) }\end{array}$ & $\begin{array}{c}\text { RA } \\
\text { J2000 }\end{array}$ & $\begin{array}{c}\text { DEC } \\
\text { J2000 }\end{array}$ & $\begin{array}{c}z \\
(\text { phot })\end{array}$ & $\begin{array}{c}B \\
\text { mag }\end{array}$ & $\begin{array}{c}V \\
\text { mag }\end{array}$ & $\begin{array}{c}R \\
\text { mag }\end{array}$ & morph \\
\hline 7 & $13: 11: 27.78$ & $-1: 20: 08.3$ & 0.193 & 20.31 & 18.50 & 17.78 & $\mathrm{E}$ \\
11 & $13: 11: 28.40$ & $-1: 20: 25.7$ & 0.202 & 21.13 & 19.19 & 18.53 & $\mathrm{E}$ \\
13 & $13: 11: 29.36$ & $-1: 20: 43.9$ & 0.180 & 20.98 & 19.43 & 18.60 & $\mathrm{Sb}$ \\
$\mathbf{1 7}$ & $13: 11: 29.99$ & $-1: 20: 29.1$ & 0.191 & 20.42 & 18.67 & 17.77 & $\mathrm{E}$ \\
19 & $13: 11: 30.18$ & $-1: 20: 52.3$ & 0.191 & 20.44 & 18.59 & 17.69 & $\mathrm{E}$ \\
$\mathbf{2 1}$ & $13: 11: 30.34$ & $-1: 20: 46.0$ & 0.191 & 20.92 & 19.28 & 18.19 & $\mathrm{E}$ \\
$\mathbf{3 9}$ & $13: 11: 35.21$ & $-1: 18: 54.9$ & 0.190 & 22.61 & 21.08 & 20.42 & $\mathrm{Sbc}$ \\
\hline
\end{tabular}

the optical counterparts to the MIR sources detected by ISOCAM (Paper I) and completed the gaps between the slitlets with bright cluster candidates. The completeness of the MOS sample is analyzed in Appendix A. It is shown that the initial selection criterion results only in a slight bias towards blue galaxies. Whenever possible, objects in the same magnitude range were arranged in each mask. The source lists of cluster members and fore-/background galaxies are given in Tables 1 and 4 , respectively. The total integration time was about 2 hours per mask, divided in 4 exposures of $30 \mathrm{~min}$ each. The disperser, ESO grism\#3, had a resolution of about 700 at $6000 \AA$. The wavelength range depended on each slitlet position on the mask. We made sure that the redshifted $4000 \AA$ break was present in all spectra. The wavelength of $\mathrm{H}_{\alpha}$ is available only for a few galaxies whereas the $[\mathrm{OII}]_{\lambda 3727}$ position is reached in most cases.

Data reduction and extraction were performed using a set of IRAF procedures written by PAD. A normalized dome flat was used as a flat-field. The wavelength calibration, based on HeAr lamp spectra, was carried out on the $2 \mathrm{D}$ spectra that were initially roughly re-positioned to a common wavelength using the info of the slitlet positions available in the headers. The spectra of several 
Table 4. Foreground and background galaxies in the MOS sample.

\begin{tabular}{|c|c|c|c|c|c|c|c|}
\hline $\begin{array}{l}\mathrm{RA} \\
\mathrm{J} 2000\end{array}$ & $\begin{array}{l}\text { DEC } \\
\text { J2000 }\end{array}$ & $\begin{array}{c}\text { ID } \\
(\mathrm{ISO})\end{array}$ & $\begin{array}{c}z \\
(\mathrm{MOS})\end{array}$ & $\begin{array}{c}B \\
\text { mag }\end{array}$ & $\begin{array}{c}V \\
\text { mag }\end{array}$ & $\begin{array}{c}R \\
\text { mag }\end{array}$ & Morph \\
\hline $13: 11: 23.95$ & $-1: 21: 46.3$ & - & 0.1330 & - & 21.55 & 21.00 & $?$ \\
\hline $13: 11: 24.58$ & $-1: 20: 04.1$ & - & 0.4816 & - & 22.65 & 21.51 & $\mathrm{Sd}$ \\
\hline 13:11:27.19 & $-1: 20: 10.5$ & 5 & $0.0862^{a}$ & 19.41 & 18.24 & 17.60 & Sab \\
\hline 13:11:27.81 & $-1: 18: 53.3$ & - & 0.3840 & - & - & 22.70 & $\mathrm{Sc}$ \\
\hline $13: 11: 28.25$ & $-1: 18: 28.1$ & - & 0.7220 & 22.94 & 21.67 & 20.91 & $\mathrm{Sd}$ \\
\hline 13:11:28.31 & $-1: 18: 32.7$ & - & 0.0130 & 21.90 & 21.34 & 20.88 & $\mathrm{E}$ \\
\hline 13:11:28.73 & $-1: 21: 43.9$ & - & 0.7900 & 21.05 & 20.97 & 20.34 & Irr \\
\hline $13: 11: 29.67$ & $-1: 17: 47.4$ & 15 & 0.3972 & 20.99 & 19.72 & 18.97 & Scd \\
\hline 13:11:30.73 & $-1: 21: 39.2$ & 23 & 0.6919 & 21.89 & 21.46 & 20.60 & Irr \\
\hline $13: 11: 33.00$ & $-1: 21: 25.1$ & - & 0.1430 & 23.16 & 22.06 & 21.43 & $\mathrm{~S} 0 / \mathrm{a}$ \\
\hline 13:11:33.69 & $-1: 19: 39.5$ & - & 0.3100 & 22.85 & 21.16 & 20.26 & - \\
\hline 13:11:34.07 & $-1: 22: 35.8$ & - & 0.4340 & - & 23.01 & 21.86 & - \\
\hline 13:11:35.18 & $-1: 20: 30.9$ & - & 0.5867 & - & 22.59 & 21.33 & $\mathrm{Sd}$ \\
\hline 13:11:35.95 & $-1: 22: 29.0$ & - & 0.2420 & 21.71 & 20.59 & 20.12 & - \\
\hline 13:11:36.40 & $-1: 22: 06.1$ & - & 0.9443 & - & 22.23 & 21.59 & - \\
\hline $13: 11: 37.37$ & $-1: 18: 37.0$ & - & $0.0825^{b}$ & 19.30 & 18.56 & 18.10 & $\mathrm{Sc}$ \\
\hline $13: 11: 38.76$ & $-1: 19: 08.2$ & - & 0.3695 & 22.05 & 20.61 & 19.97 & $\mathrm{Sd}$ \\
\hline $13: 11: 41.83$ & $-1: 19: 48.1$ & - & 0.1030 & 21.19 & 19.94 & 19.35 & - \\
\hline
\end{tabular}

Notes: ${ }^{a}$ [TCG90] 217; the redshift of 0.2153 as given in Teague et al. (1990), is presumably wrong. ${ }^{b}$ [TCG90] 006; the redshift of 0.1826 , as given in Teague et al. (1990), is presumably wrong.

spectrophotometric standard stars were obtained with the same grism and $5^{\prime \prime}$ wide long-slits. A few objects were observed through different masks with their corresponding slitlets put at various locations. We could hence check the relative accuracy of the flux calibration in the MOS field, which turns out to be better than $20 \%$.

Redshifts were determined from the average value of individual emission and absorption lines with a stronger weight given to emission lines. For spectra with very low signal to noise, no obvious lines could be identified; instead the wavelength of the $4000 \AA$ decrement was used. The redshift of thirty galaxies could be compared with that measured by Teague et al. (1990). They are identical (within 1\%) for all of them, but two ${ }^{3}$. Our redshifts are listed in Tables 1 and 4 .

Line measurements were performed with two techniques: manually, with the Gaussian fitting provided by the "splot" procedure in IRAF, and semi-automatically with a purposely written program (MORPHS collaboration, Dressler et al. 1999). They agree well with each other, although the manual technique underestimates the

${ }^{3}$ [TCG90] 006 and [TCG90] 217 have a redshift of resp. 0.0825 and 0.0862 instead of 0.1826 and 0.2153 as reported in Teague et al. (1990). Given the number of spectral features, we believe our redshifts are the correct ones. equivalent widths by $15-20 \%$. This is due to systematic differences in the adopted level of the continuum. In order to compare our results with those of the MORPHS group, we decided to base our spectral classification and analysis on the measurements obtained with the semi-automatic method. For galaxies observed through different masks, we retained the spectra with the highest signal to noise. The spectrophotometric data of our MOS run are listed in Table 5 .

\section{Results}

\subsection{Cluster membership and velocity distribution}

The histograms shown in Fig. 1 collect all redshift information available for galaxies lying in the $5^{\prime} \times 7^{\prime}$ field towards Abell 1689. Spectroscopic redshifts mainly come from our MOS survey and from the spectroscopic survey by Teague et al. (1990). In addition, Dye et al. (2001) determined the photometric redshifts of several hundreds of galaxies observed through an optimized set of narrowband and broad-band filters. Not surprisingly, all histograms show a strong concentration of galaxies in the redshift range 0.17-0.22, presumably members of Abell 1689. The peak is at $z_{\text {spectro }}=0.184$. While the large dispersion in the redshift distribution based on the photometric 
Table 5. Spectrophotometric data of cluster members from the MOS sample.

\begin{tabular}{|c|c|c|c|c|c|c|c|}
\hline $\begin{array}{l}\text { ID } \\
(\mathrm{MOS})\end{array}$ & $\begin{array}{l}\text { ID } \\
\text { (ISO) }\end{array}$ & $\begin{array}{c}\text { ID } \\
\text { (mask) }\end{array}$ & $\begin{array}{l}\text { flux }\left([\mathrm{OII}]_{\lambda 3727}\right) \\
\operatorname{erg} \mathrm{cm}^{-2} \mathrm{~s}^{-1}\end{array}$ & $-E Q W\left([\mathrm{OII}]_{\lambda 3727}\right)$ & $\begin{array}{c}E Q W\left(\mathrm{H}_{\delta}\right) \\
\AA\end{array}$ & Class & Comments \\
\hline 1 & - & MOS5:17 & - & - & - & $\mathrm{k}$ : & poor \\
\hline 2 & - & $\operatorname{MOS} 2: 9$ & $4.67 \pm 0.19$ & $32.8 \pm 1.7$ & $4.8: \pm 1.0$ & $\mathrm{e}(\mathrm{c})$ & $\mathrm{H}_{\beta}=-8.2, \mathrm{OIII}=-14.0$ \\
\hline 3 & - & MOS3:20 & - & - & - & $?$ & poor \\
\hline 4 & - & MOS1:12 & - & - & $3.8: \pm 2.1$ & $\mathrm{k}+\mathrm{a}:$ & poor \\
\hline 5 & - & MOS1:6 & - & - & - & $\mathrm{k}$ : & $\mathrm{OIII}=-1.5 ?$ \\
\hline 6 & - & MOS3:17 & - & - & - & $\mathrm{k}:$ & poor \\
\hline 7 & 3 & MOS5:11 & $0.60 \pm 0.32$ & $3.9 \pm 1.6$ & - & $\mathrm{k}(\mathrm{e})$ & \\
\hline 9 & - & MOS4:17 & - & - & $3.8 \pm 1.4$ & $\mathrm{k}+\mathrm{a}$ & \\
\hline 10 & - & $\operatorname{MOS} 1: 7$ & - & - & - & $\mathrm{k}:$ & \\
\hline 11 & .. & MOS1:36 & $2.73 \pm 0.28$ & $8.6 \pm 1.0$ & $2.3 \pm 0.7$ & $\mathrm{e}(\mathrm{c}:)$ & $\mathrm{H}_{\beta}=-2.9, \mathrm{OIII}=-9.4, \mathrm{OIII} 2=-2.7$ \\
\hline 12 & - & MOS3:19 & - & - & - & $?$ & poor \\
\hline 13 & - & $\operatorname{MOS} 2: 6$ & - & - & - & $?$ & poor \\
\hline 14 & 4 & MOS4:10 & $1.85 \pm 0.27$ & $18.8 \pm 3.3$ & - & $\mathrm{e}(\mathrm{c})$ & $\mathrm{H}_{\beta}=-3.6$ \\
\hline 15 & - & $\operatorname{MOS} 5: 3$ & - & - & - & $\mathrm{k}:$ & \\
\hline 16 & 6 & MOS5:9 & $2.36 \pm 0.11$ & $60.0 \pm 3.0$ & - & $\mathrm{e}(\mathrm{b})$ & $\mathrm{H}_{\beta}=-12.8, \mathrm{OIII}=-44.4$ \\
\hline 17 & - & MOS5:8 & - & - & - & $\mathrm{k}$ & \\
\hline 20 & - & MOS2:22 & - & - & - & $\mathrm{k}:$ & poor \\
\hline 21 & 8 & MOS5:10 & - & - & - & $\mathrm{k}$ & \\
\hline 22 & - & MOS4:21 & - & - & - & $\mathrm{k}:$ & poor \\
\hline 23 & 10 & MOS2:15 & $3.05 \pm 0.23$ & $10.4 \pm 1.0$ & $2.1: \pm 0.8$ & $\mathrm{e}(\mathrm{a})+$ & $<\mathrm{H} \theta+\mathrm{H} \eta+\mathrm{H} \zeta>/ 3=5.1, \mathrm{H}_{\delta}$ prob.higher, $\mathrm{H}_{\beta}=-0.8$ \\
\hline 24 & - & $\operatorname{MOS} 1: 4$ & - & $<3.3$ & - & $\mathrm{k}:(\mathrm{e}:)$ & $\mathrm{H}_{\beta}=2.0$ \\
\hline 25 & - & MOS3:13 & - & - & - & $\mathrm{k}:$ & \\
\hline 26 & - & MOS4:19 & - & - & - & $?$ & \\
\hline 27 & - & MOS4:18 & - & - & $3.0: \pm 1.3$ & $\mathrm{k}+\mathrm{a}:$ & \\
\hline 28 & 12 & MOS1:29 & - & - & $5.5 \pm 0.7$ & $\mathrm{k}+\mathrm{a}$ & \\
\hline 29 & 14 & MOS2:13 & - & - & - & $\mathrm{k}$ & \\
\hline 30 & .. & MOS $2: 25$ & - & - & $2.1 \pm 0.9$ & $\mathrm{k}$ & \\
\hline 31 & - & MOS5:21 & - & - & - & $\mathrm{k}:$ & poor \\
\hline 32 & - & MOS4:14 & - & - & $6.7: \pm 2.4$ & $\mathrm{k}+\mathrm{a}:$ & poor \\
\hline 33 & 16 & MOS4:11 & - & - & - & $\mathrm{k}$ : & poor \\
\hline 34 & - & MOS5:5 & - & - & - & $\mathrm{k}$ & \\
\hline 35 & - & MOS5:12 & $0.23: \pm 0.09$ & $7.4: \pm 2.3$ & - & $\mathrm{k}(\mathrm{e}:)$ & \\
\hline 36 & .. & MOS1:35 & $0.53: \pm 0.18$ & $2.1: \pm 1.1$ & $4.2: \pm 1.7$ & $\mathrm{k}+\mathrm{a}:(\mathrm{e})$ & NII in em? \\
\hline 37 & 18 & MOS4:12 & - & $<5.4$ & $1.8 \pm 0.7$ & $\mathrm{k}(\mathrm{e}: \mathrm{:})$ & \\
\hline 38 & - & MOS4:3 & - & - & $2.8 \pm 1.1$ & $\mathrm{k}+\mathrm{a}:$ & \\
\hline 42 & 24 & MOS1:11 & - & - & - & $\mathrm{k}$ & \\
\hline 43 & 26 & MOS1:20 & - & - & - & $\mathrm{k}$ & \\
\hline 44 & - & MOS5:16 & - & - & - & $\mathrm{k}:$ & \\
\hline 45 & .. & MOS3:22 & - & - & - & $\mathrm{k}:$ & poor \\
\hline 46 & 30 & MOS4:16 & - & - & - & $\mathrm{k}$ : & \\
\hline 47 & - & $\operatorname{MOS} 2: 4$ & - & - & - & $\mathrm{k}$ & \\
\hline 48 & - & MOS3:5 & $1.50 \pm 0.17$ & $46.2 \pm 7.0$ & - & $\mathrm{e}(\mathrm{b})$ & $\mathrm{H}_{\beta}=-6.7, \mathrm{OIII}=-11.4, \mathrm{H}_{\alpha}=-43.1$ \\
\hline 49 & 31 & MOS1:24 & $2.48 \pm 0.26$ & $7.1 \pm 0.9$ & $5.5 \pm 0.8$ & $\mathrm{e}(\mathrm{a})$ & $<\mathrm{H} \theta+\mathrm{H} \eta+\mathrm{H} \zeta>/ 3=5.2$ \\
\hline 50 & - & MOS1:25 & $\begin{array}{c}2.90 \pm 0.20 \\
-\end{array}$ & $\begin{array}{ll}1 \\
-1\end{array}$ & $\begin{array}{ll}0.0 \\
-\end{array}$ & $?$ & very poor \\
\hline 51 & 32 & MOS1:18 & - & - & $1.1 \pm 0.8$ & $\mathrm{k}$ & \\
\hline 52 & 33 & MOS2:16 & - & - & $2.0 \pm 0.5$ & $\mathrm{k}$ & \\
\hline 53 & .. & MOS1:32 & - & - & - & $\mathrm{k}$ & \\
\hline 54 & 35 & MOS1:13 & $1.90 \pm 0.17$ & $8.1 \pm 0.8$ & $3.5 \pm 0.7$ & $\mathrm{e}(\mathrm{a})+$ & $<\mathrm{H} \theta+\mathrm{H} \eta+\mathrm{H} \zeta>/ 3=5.5, \mathrm{Ha}+\mathrm{NII}=-3.1$ \\
\hline 55 & 37 & MOS1:27 & $1.00 \pm 0.30$ & $3.9 \pm 1.0$ & $2.8 \pm 1.4$ & $\mathrm{e}(\mathrm{a})+$ & $<\mathrm{H} \theta+\mathrm{H} \eta+\mathrm{H} \zeta>/ 3=5.7, \mathrm{Ha}+\mathrm{NII}=-13$ \\
\hline 56 & .. & MOS1:30 & - & - & $2.3: \pm 1.6$ & $\mathrm{k}(\mathrm{e}:)$ & $\mathrm{H}_{\alpha}=-2.3 ?$ \\
\hline 57 & - & MOS3:11 & - & - & - & $\mathrm{k}$ : & poor \\
\hline 58 & .. & MOS1:2 & $0.18: \pm 0.07$ & $2.5: \pm 0.9$ & $3.4 \pm 1.2$ & $\mathrm{k}+\mathrm{a}(\mathrm{e}:)$ & \\
\hline 59 & 40 & MOS3:9 & - & - & - & ? & very poor \\
\hline 60 & .. & MOS4:24 & - & - & - & $\mathrm{k}+\mathrm{a}:$ & earl Bals v. str (to be meas) \\
\hline 61 & 41 & MOS2:14 & - & - & $3.5 \pm 0.8$ & sey1: & $\mathrm{OIII}=-5.1$, broad $\mathrm{Hb}$ em $=-3.7$, broad $\mathrm{Ha}+\mathrm{NII} * 2=-28.4$ \\
\hline 62 & - & MOS2:19 & - & - & - & $?$ & poor \\
\hline 63 & .. & MOS3:3 & .. & .. & - & $\mathrm{e}(\mathrm{c})$ & sky on OII,no $\mathrm{H}_{\delta}, \mathrm{OIII}=-16.8, \mathrm{H}_{\beta}=-6.5, \mathrm{H}_{\alpha}=-9.1$ \\
\hline 64 & - & $\operatorname{MOS} 1: 5$ & - & - & - & $\mathrm{k}:$ & poor \\
\hline 65 & .. & MOS4:25 & - & - & - & $\mathrm{k}$ & no \\
\hline 66 & - & MOS2:17 & - & - & $2.5 \pm 1.0$ & $\mathrm{k}$ & \\
\hline 67 & .. & $\operatorname{MOS} 3: 4$ & - & - & - & $\mathrm{k}(\mathrm{e})$ & $\mathrm{NII} 2=-4.8$ \\
\hline 68 & .. & MOS3:21 & - & - & $4.1: \pm 2.2$ & $\mathrm{k}+\mathrm{a}:$ & \\
\hline 69 & 43 & MOS2:10 & .. & .. & $2.9 \pm 1.0$ & $\mathrm{e}(\mathrm{c})$ & $\mathrm{H}_{\beta}=1.4, \mathrm{Ha}>-4.2, \mathrm{NII}^{*} 2$ \\
\hline 70 & .. & MOS2:26 & .. & .. & - & $\mathrm{k}$ & \\
\hline 71 & .. & MOS5:20 & .. & .. & - & $\mathrm{k}$ & $\mathrm{H}_{\beta}=1.9$ \\
\hline 72 & 45 & MOS3:16 & .. & .. & - & $\mathrm{e}(\mathrm{b})$ & $\mathrm{OIII}=-11.7, \mathrm{H}_{\beta}=-15.4, \mathrm{Ha}>-42.7, \mathrm{NII} 2$ \\
\hline 73 & .. & MOS4:23 & .. & .. & - & k: & \\
\hline 74 & .. & $\operatorname{MOS} 1: 21$ & .. & .. & - & $?$ & poor \\
\hline
\end{tabular}

Notes: ".." means that the $[\mathrm{OII}]$ or $\mathrm{H}_{\delta}$ line is outside the MOS wavelength range. The equivalent width of a number of some other lines are indicated in the comments. 


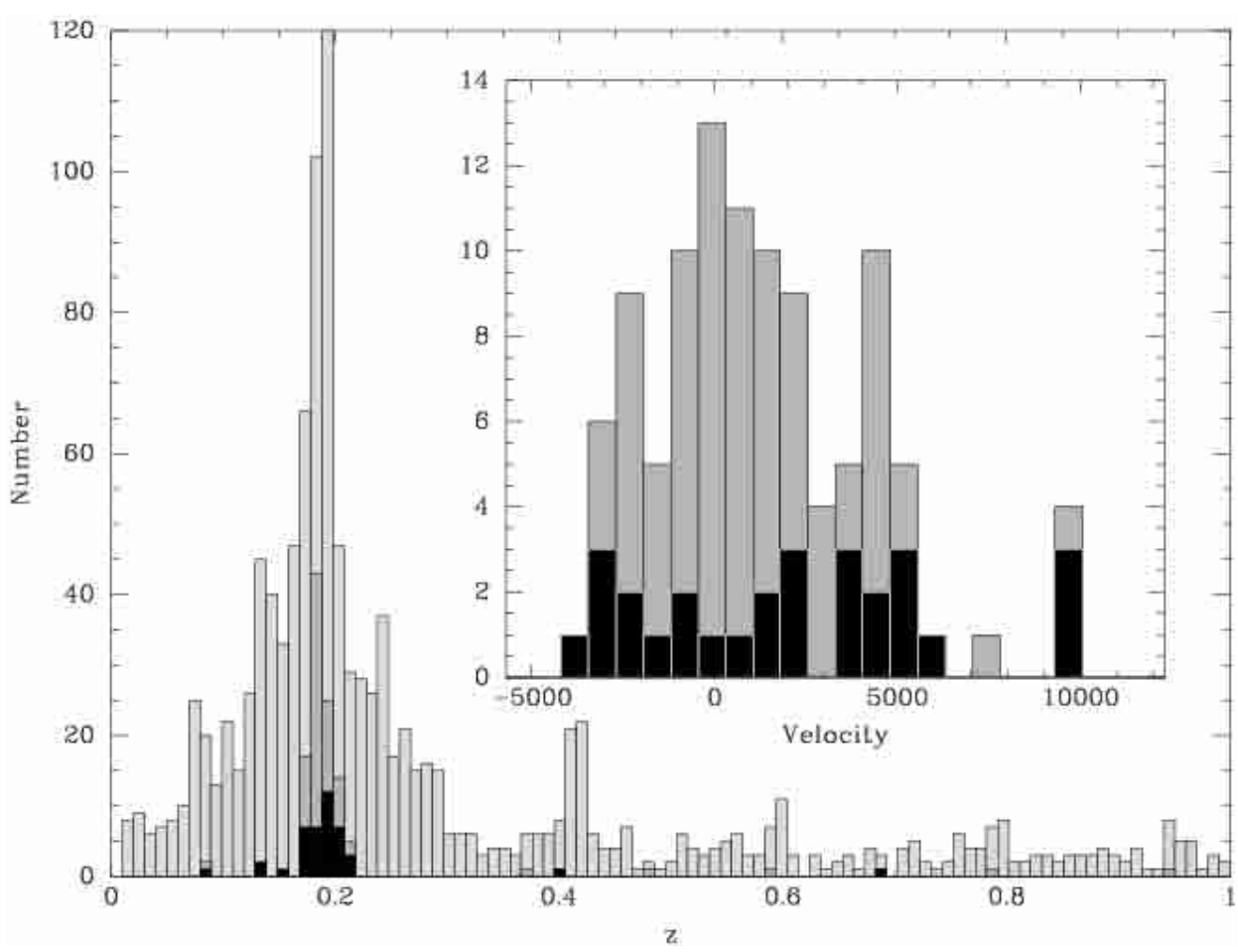

Fig. 1. Redshift histogram towards Abell 1689 in the redshift range $z=0-1$. Several samples are included: optically selected galaxies with photometric redshifts from the catalog by Dye et al. (2001) (light shaded bars) or spectroscopic redshifts from our MOS survey and from the literature (shaded bars); ISOCAM-selected galaxies with either spectroscopic or photometric redshifts (dark shaded bars). The inset shows a close up on the redshift range $z=0.17-0.22$, that considered for cluster membership. Only ISOCAM-selected galaxies with well determined spectroscopic redshifts are included in the latter plot. The $x$-axis is scaled in $\mathrm{km} \mathrm{s}^{-1}$.

technique results from the uncertainty of the method, the substantial velocity spread indicated by the spectroscopic observations for galaxies related to A1689 is real (see inset in Fig. 1). It reflects both the richness of the cluster and its complex velocity structure. Applying a multiscale analysis, Girardi et al. (1997) distinguished three distinct groups in A1689 that overlap spatially but are well separated in velocity. Hence, what we see in projection towards the central region of this cluster (see Fig. 2) is probably composed of different subclumps merging along the line of sight.

Figure 1 indicates that the ISOCAM-detected galaxies also show a well-defined peak in correspondence with the cluster baricentric velocity. Actually, more than $80 \%$ of the ISOCAM sources have redshifts between $0.17-0.22$. This prominent excess of mid-infrared emitters related to the cluster is real and does not result from selection effects in the optical follow-up (see Paper I). However, the velocity distribution of ISOCAM sources related to A1689 is flatter than that of the bulk of the cluster population (see inset in Fig. 1). This result is actually expected. We will show later that most MIR emitters are associated with spiral and emission-line galaxies. Studies of nearby clusters indicate a larger velocity dispersion for this population than for the early-type galaxies (e.g. Biviano et al. 1997).

The observation of a well defined peak in the redshift histogram at $z=0.17-0.22$ both in the optically selected and mid-infrared selected samples provide a redshift- based criteria to assess the cluster membership. In the following analysis, we consider as cluster members at large all galaxies in the above-mentionned redshift range. They might be long standing members, galaxies just falling in the cluster or belonging to merging sub-clusters. Their excess of infrared activity as detected by ISOCAM might trace the broad cluster environment, or the cluster itself. A detailed analysis of the precise role of the environment goes beyond the limits of this study for lack of statistics and field coverage. Our survey only extends out to a clustercentric radius of $0.5 \mathrm{Mpc}$, therefore inferring a radial dependence of the properties of the cluster members is not possible.

\subsection{Properties of the optically selected cluster members}

In this section we present the photometric, spectroscopic and morphological properties of galaxies in our spectroscopic sample, comparing them when possible with the properties of lower and higher redshift clusters.

\subsubsection{The optical subsamples}

The 74 cluster members in our MOS survey are listed in Table 1. In the following analysis we include another 17 galaxies for which redshifts are available in the 


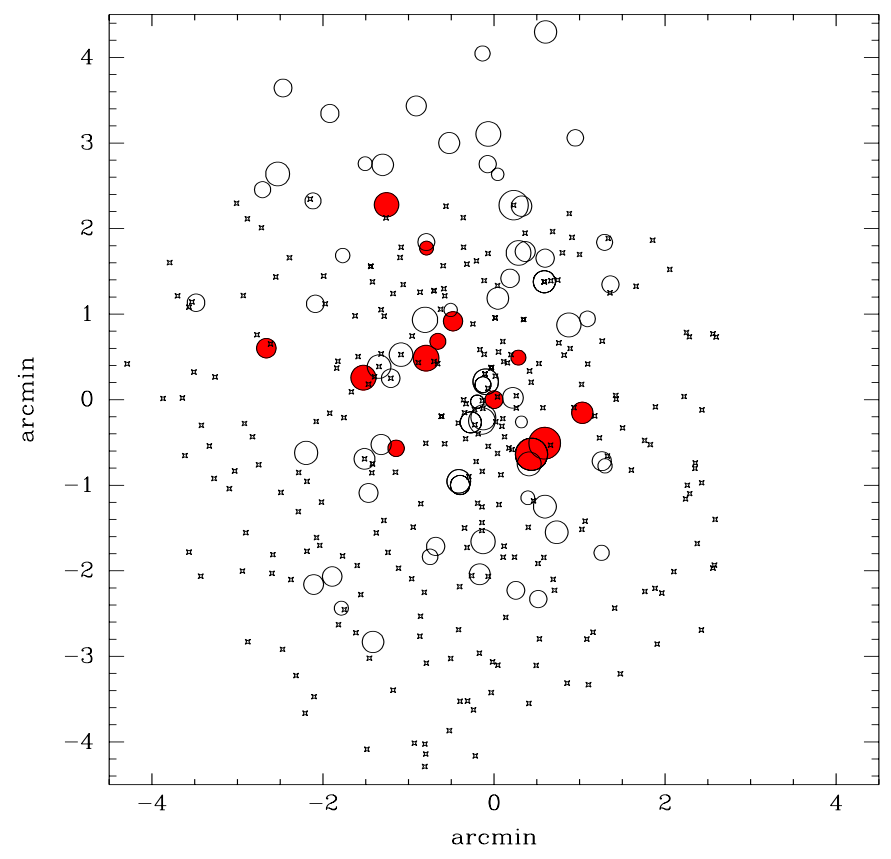

Fig. 2. Spatial and velocity distribution of cluster members. Photometrically confirmed members are shown with the small stars. Spectroscopically confirmed members are shown with circles the size of which is proportional to their velocity in the cluster. Finally, the filled (red) circles correspond to the ISOCAM $15 \mu \mathrm{m}$ sources.

literature (Teague et al. 1990). These are listed in Table 2. We also make use of the 167 galaxies with an $\mathrm{R}$ band magnitude brighter than 22 , which have a photometric redshift determined by Dye et al. (2001) compatible with a cluster membership. Those detected by ISOCAM are listed in Table 3. For reference, we indicate in Table 4 the foreground and background galaxies found in our MOS survey.

\subsubsection{Blue fraction}

The value of the blue fraction, $f_{\mathrm{B}}$, in Abell 1689, i.e. the proportion of galaxies bluer than the color-magnitude relation, is controversial. In their original article, Butcher $\&$ Oemler (1984) computed a value of $f_{\mathrm{B}}=0.09 \pm 0.03$. However, later on Gudehus \& Hegyi (1991) estimated a lower value for $f_{\mathrm{B}}(0.05 \pm 0.06)$ consistent with the blue fraction measured in local clusters. Recently, $f_{\mathrm{B}}$ was revised again and raised to: $0.093 \pm 0.019$ (Margoniner \& de Carvalho 2000) and even $0.191 \pm 0.015$ (Margoniner et al. 2001). Such a discrepancy can be due to the many observational biases, the fuzziness of the definition of the blue fraction (i.e. the choice of the color index, luminosity range, $k$-correction model, location of the color-magnitude diagram etc.) and especially the background subtraction.

We estimated $f_{\mathrm{B}}$ using our dataset of confirmed cluster members (see details in Appendix B). Our computed value is 1.5-2 times that originally derived by Butcher \& Oemler (1984) for this cluster and appears to be much higher than in the local rich clusters studied by these authors. Large blue fractions have also been reported in other clusters at $z=0.2$ (e.g. in Abell 115, Metevier et al. 2000), but there is clearly a large spread in $f_{\mathrm{B}}$ among rich clusters at this redshift (Smail et al. 1998).

\subsubsection{Spectral and morphological classification}

We classified all spectra using the set of rules proposed by Dressler et al. (1999), which are based on the rest-frame equivalent widths of the $[\mathrm{OII}]_{\lambda 3727}$ emission line and the $\mathrm{H}_{\delta}$ absorption line ${ }^{4}$. No spectral type could be assigned to the spectra of 8 cluster members due to their low signal to noise.

Representative spectra for each class are presented in Fig. 3 and the histogram of the spectral types is shown in Fig. 4. At least $50 \%$ of the spectroscopically confirmed members have spectra of type "k" - they show no emission lines and only weak Balmer absorption lines - and are hence typical of passive early type galaxies. The " $\mathrm{k}+\mathrm{a}$ " type, characterized by the presence of strong Balmer absorption lines typical of post-starburst/post-starforming galaxies, accounts for 10 to $15 \%$ of our spectroscopic sample. This fraction is significantly higher than that typically estimated in nearby clusters $(\sim 1 \%$ according to Dressler 1987). A direct comparison with higher redshift clusters is hindered by the different completeness as a function of galaxy magnitude of the various spectroscopic samples. In clusters at $z \sim 0.5$, the $\mathrm{k}+\mathrm{a}$ fraction was found to be about 20\% (MORPHS collaboration, Dressler et al. 1999; Poggianti et al. 1999). Using a sample with a mean redshift lower than the MORPHS, the CNOC1 group (Balogh et al. 1999) estimated a much lower proportion of post-starburst galaxies, less than 5\%. On the other hand, Abraham et al. (1996) counted in Abell 2390 a proportion of $\mathrm{H}_{\delta}$-strong galaxies as high as $23 \%$, in good agreement with Abell 1689 where the $\mathrm{H}_{\delta}$-strong objects $(\mathrm{k}+\mathrm{a}$ 's and e(a)'s) amount to about 20\%. The two clusters are situated at the same redshift.

In total about $20 \%$ of the cluster members in our sample are emission line galaxies showing some level of star formation in the optical. Conversely, most of the galaxies in the cluster classified as "blue" - those responsible for the photometric Butcher-Oemler effect - exhibit emission lines that are indicative of a current star formation.

The spectroscopic sample includes only one Seyfert 1 galaxy, identified by the broad $\mathrm{H}_{\alpha}$ emission line. Usual diagnostics to distinguish Seyfert 2/LINERs from HII regions (Veilleux \& Osterbrock 1987) could not be applied because the required $\mathrm{H}_{\alpha}$ hydrogen, [NII] nitrogen and [SII] sulfur lines were outside the spectral

\footnotetext{
${ }^{4}$ We have introduced an additional spectral class, the " $\mathrm{k}(\mathrm{e})$ " type, which represents spectra similar to the k-type but with signs of at least one very weak emission line. The definition of the "e(a)" class was also slightly modified, as indicated and justified in Sect. 3.3.4. Finally, seven galaxies for which the $[\mathrm{OII}]_{\lambda 3727}$ line was outside the MOS wavelength range were classified based on their Hydrogen Balmer lines.
} 


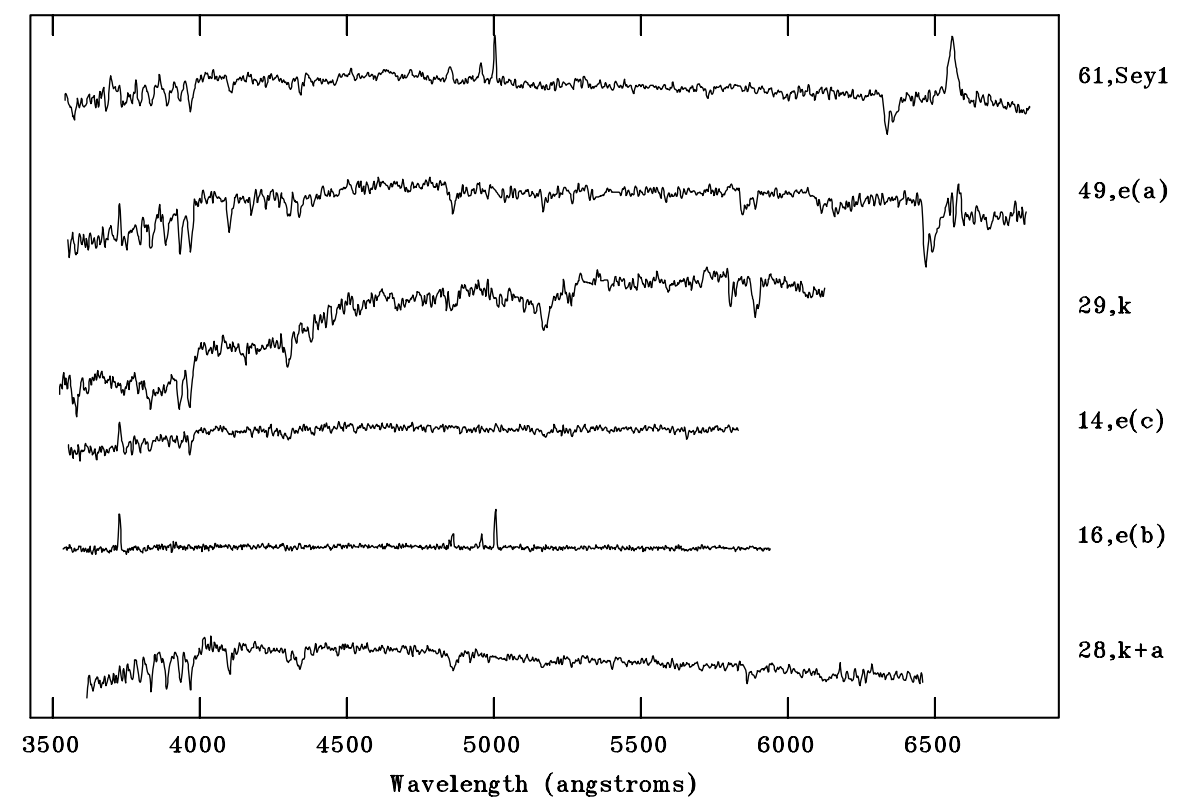

Fig. 3. Representative optical spectra of cluster members with different spectral types. All spectra except the bottom one belong to $L W 3$-detected galaxies. From bottom to top, spectra are ordered by increasing $15 \mu \mathrm{m}$ flux. The MOS ID and spectral type are indicated to the right.

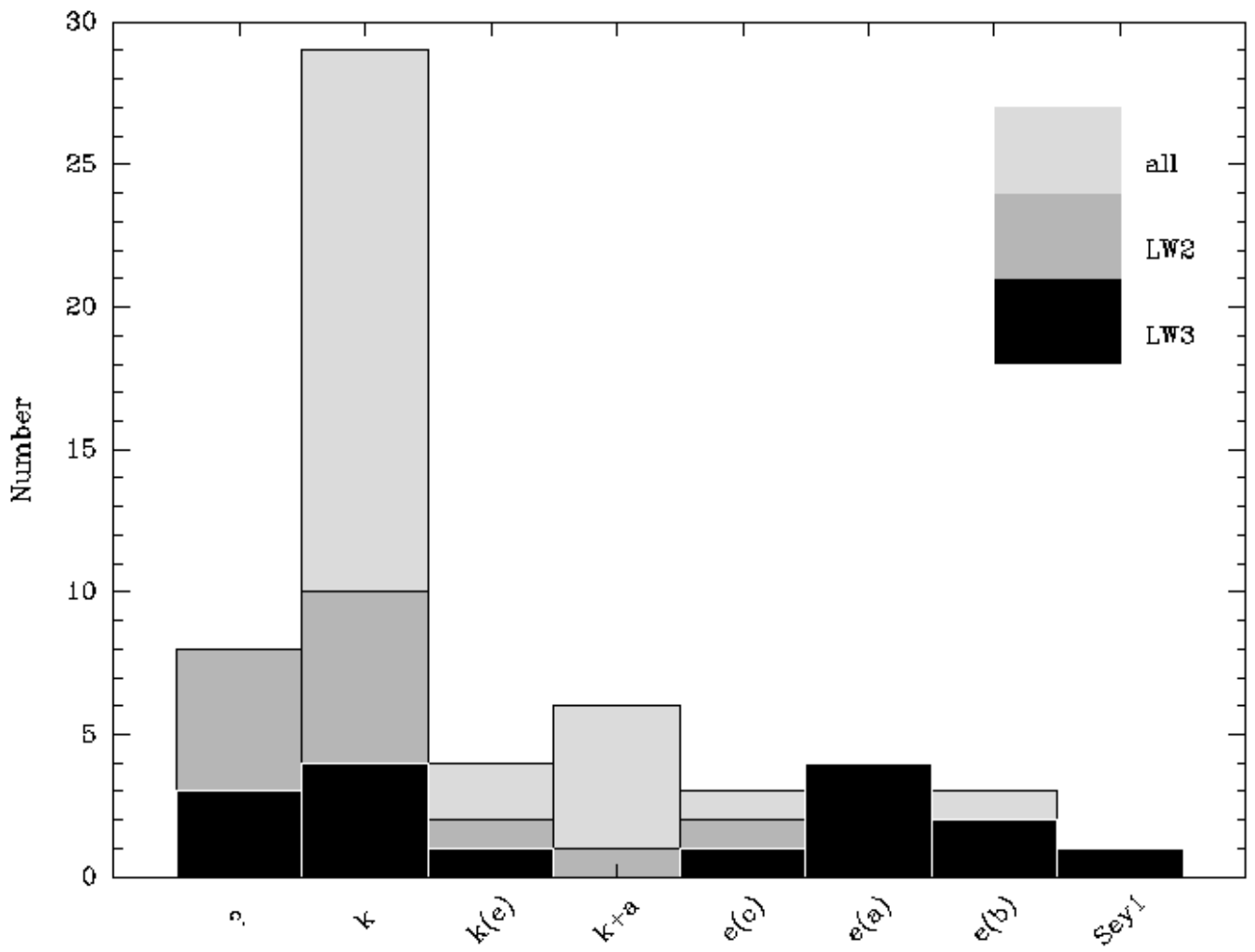

Fig. 4. Spectral type histogram of all MOS (light shaded), $6.75 \mu \mathrm{m}$ detected (shaded, $L W 2$ ) and $15 \mu \mathrm{m}$ detected (dark shaded, $L W 3$ ) cluster members in the ISOCAM field of view. Note that almost all $L W 3$ sources have also been detected in the $L W 2$ filter. The unknown class "?" either includes galaxies for which the signal to noise was too low to determine a redshift or ISOCAM sources missed by our MOS survey.

range of most galaxies. Instead, we have used the recent diagnostics of Rola et al. (1997), based on lines in the blue only. We found that no AGN activity was required to explain the optical spectra of the emission line galaxies but the Seyfert 1.
Finally, we note that all spectral types are evenly distributed throughout our field of view. Star-forming galaxies are present even at low projected clustercentric radii. The field-of-view and the sub-clustering and related projection effects (see Sect. 3.1) are likely to be the reasons 
why no clear radial trend is actually observed in any of the galactic properties (optical color, spectral characteristics, morphology).

The morphologies of galaxies towards Abell 1689 were derived and kindly provided to us by the MORPHS group from HST WFPC2 images, and are included in Table 1. A description of the classification method may be found in Smail et al. (1997). The fraction of E-type galaxies does not change if photometric or spectroscopic members are considered, showing that our MOS survey is not biased towards/against any particular Hubble type of galaxies. Within our spectroscopic sample, the majority (60\%) of the members have morphologies typical of early type galaxies (ellipticals and lenticulars). We find a quite large fraction of lenticulars (about 35\%) and an even larger fraction of spirals (at least $40 \%)^{5}$. The latter is significantly larger than the spiral fraction in rich clusters at $z=0$ (e.g. see Fig. 9 in Fasano et al. 2000).

To conclude, for many aspects, the galaxies in Abell 1689 - despite their low redshift - seem to have different optical properties than those of galaxies in typical rich clusters in the nearby universe. Several of the evolutionary trends associated with the Butcher-Oemler effect, i.e. a change in the photometric, spectroscopic and morphological properties of the cluster members, may already be seen in this $z=0.18$ cluster.

\subsection{Properties of the MIR selected cluster members}

Abell 1689 is the first cluster at moderate distance that has been mapped in the mid-infrared regime with enough sensitivity to compute statistics on the properties of the MIR emitters. In the following, we will focus our analysis on the mid-infrared emitters in the cluster and discuss more in detail the IR side of the Butcher-Oemler effect that has been reported in Paper I.

\subsubsection{The MIR subsamples}

The ISOCAM cluster sample consists of 30 galaxies detected at $6.75 \mu \mathrm{m}$ with a mean $L W 2$ flux of $0.23 \mathrm{mJy}$ and 16 detected at $15 \mu \mathrm{m}$ with a mean $L W 3$ flux of $0.57 \mathrm{mJy}^{6}$. Among them, 15 sources have been detected at both wavelengths. For most MIR sources, the cluster membership has been assessed using spectroscopic redshifts. Seven MIR sources have been missed in our MOS survey and were considered as cluster members based on their photometric redshift only. They are listed in Table 3.

\footnotetext{
${ }^{5}$ The fractions do not change if only galaxies with $M_{V}<$ -20 are included. This magnitude cut is the same as the one applied in other studies of galaxy morphologies, including Fasano et al. (2000). The cluster area sampled here is similar to the area used in these other works (the central $\mathrm{Mpc}^{2}$ ). A comparison of the morphological fractions in A1689 with those given by these other authors is therefore justified.

6 The galaxy ISO \#28 which, with a photometric redshift of 0.15 , was considered as a possible cluster member in Paper I has not been included here.
}

Five $L W 3$ sources have a low signal to noise at $15 \mu \mathrm{m}$ but were included in the statistics because the reliability of the detection was confirmed by the discovery of a strong $6.75 \mu \mathrm{m}$ counterpart (see Paper I). The $L W 2$ sources towards the crowded central regions suffer severe blending problems. They were deblended into several sources and their relative $L W 2$ fluxes were assigned based on the relative luminosities of the optical counterparts candidates (Paper I).

\subsubsection{Luminosity and color}

The $R$-band magnitude and $B-R$ color distributions of the cluster MIR emitters are shown in Fig. 5 and Fig. 6, superimposed on the histograms of the optically selected cluster members. Clearly, the $L W 2$ sources correspond to the brightest and reddest galaxies in the cluster while the $L W 3$ sources have a slightly flatter optical luminosity function and moreover span a large color range. These results may be better visualized in the color-magnitude diagram shown in Fig. 7 where galaxies detected at 6.75 and $15 \mu \mathrm{m}$ are indicated by resp. a square and a circle. The size of each symbol is proportional to the $L W 3 / L W 2$ flux ratio. The majority of the bright blue galaxies in the cluster - those responsible for the photometric BO effect - turn out to have a large $15 \mu \mathrm{m}$ to $6.75 \mu \mathrm{m}$ flux ratio. In the magnitude range $18.5-19.5$ only blue galaxies are detected through the $L W 3$ filter.

\subsubsection{Morphology}

HST morphologies are available for almost all ISOCAM sources. Figure 8 displays the optical images of the MIR emitters. These flux-calibrated images are all shown with the same spatial and intensity scale and are ordered according to the MIR color $L W 3 / L W 2$.

First of all, we note a correlation between the MIR color and the size and luminosities of the optical counterparts, sources with a low $L W 3 / L W 2$ ratio being generally bigger in size and more luminous in the optical. About half of the $L W 2$ sources have morphologies characteristic of early type galaxies (E and S0s). Their $L W 2$ light is expected to be contaminated by the large integrated stellar photospheric emission. The galaxies with the highest $L W 3 / L W 2$ ratio and the majority (75\%) of the $L W 3$ emitters are spiral galaxies. This is expected since most of the emission at rest-frame $12.5 \mu \mathrm{m}$ arises from star-forming regions which are prevalent in late-type galaxies. We note however that a few galaxies -3 galaxies or $19 \%$, which is already significant - of $L W 3$ sources have morphologies typical of elliptical galaxies, and 1 is an S0. Among the galaxies with a high $L W 3 / L W 2$ ratio, several of them have clearly a disturbed morphology: for example, perturbed external disks (ISO source \#37, the strongest MIR emitter in the sample), isophote twisting (ISO\#4), weak tidal tail (ISO\#41) or interacting galaxies (ISO\#6 and, possibly, \#39). Morphologically peculiar 


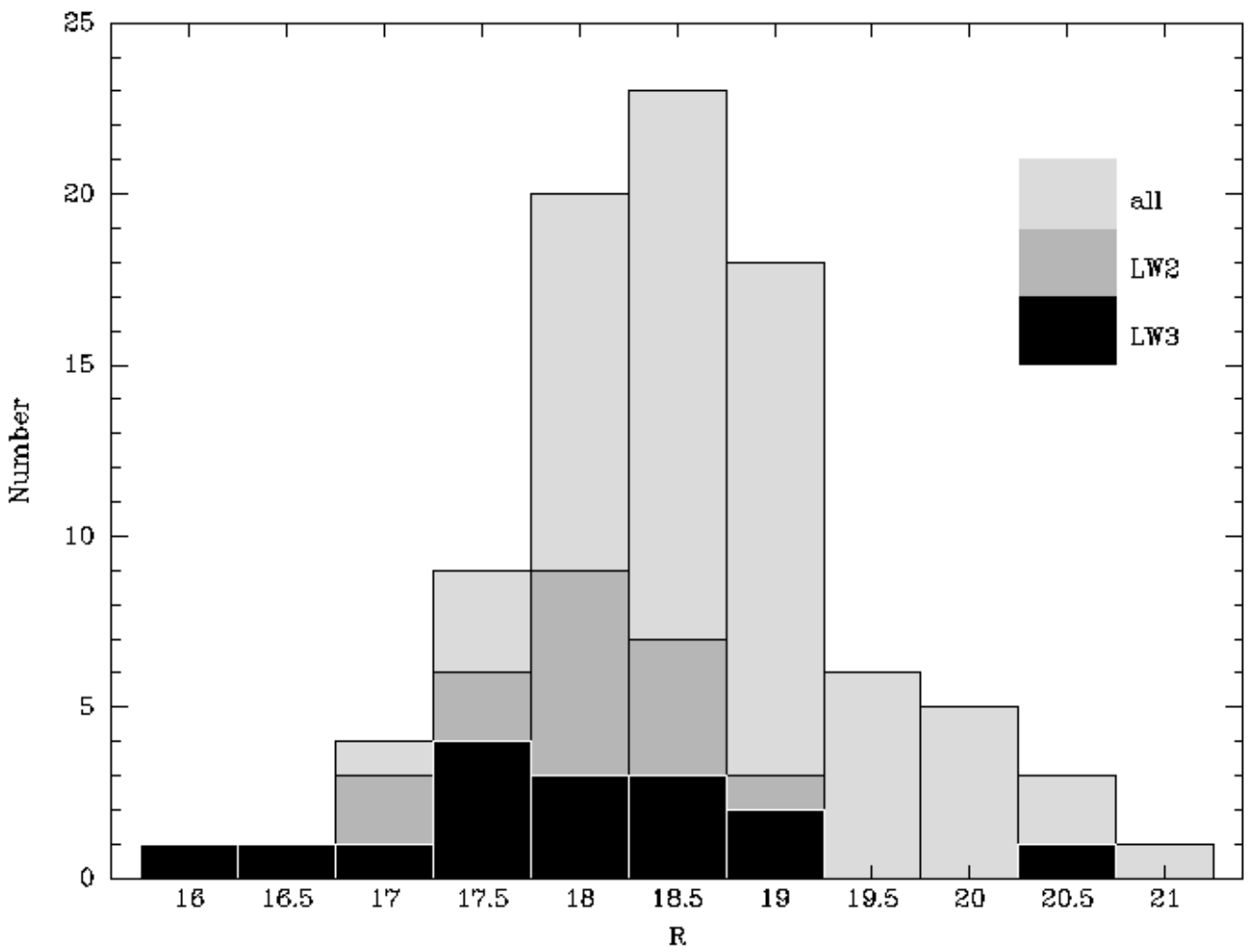

Fig. 5. $R$-band magnitude histogram of spectroscopically confirmed (light shaded), $6.75 \mu \mathrm{m}$ detected (shaded, $L W 2$ ) and $15 \mu \mathrm{m}$ detected (dark shaded, $L W 3$ ) cluster members within the ISOCAM field of view.

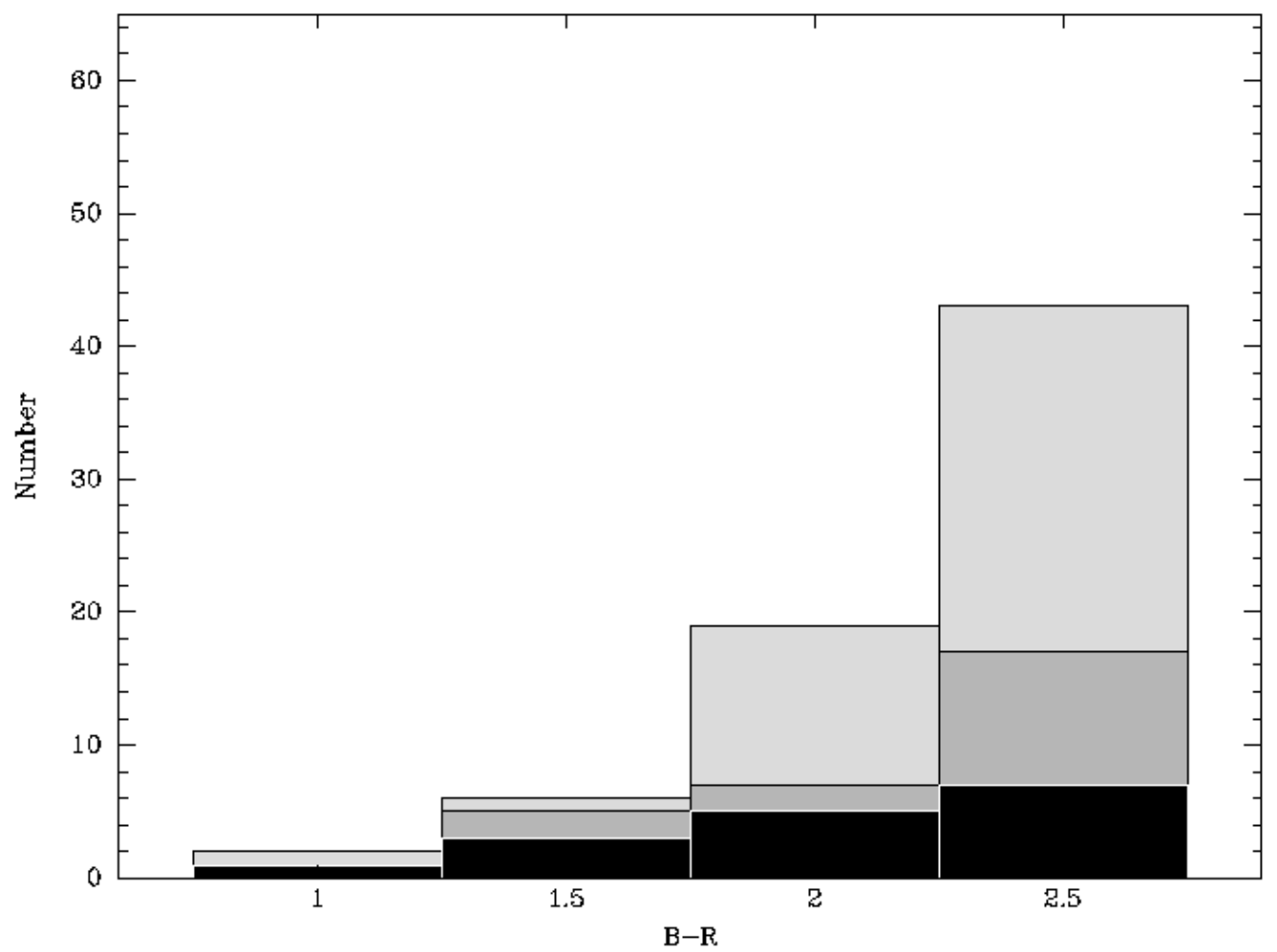

Fig. 6. $B-R$ color index histogram of spectroscopically confirmed (light shaded), $6.75 \mu \mathrm{m}$ detected (shaded, $L W 2)$ and $15 \mu \mathrm{m}$ detected (dark shaded, $L W 3$ ) cluster members within the ISOCAM field of view. 


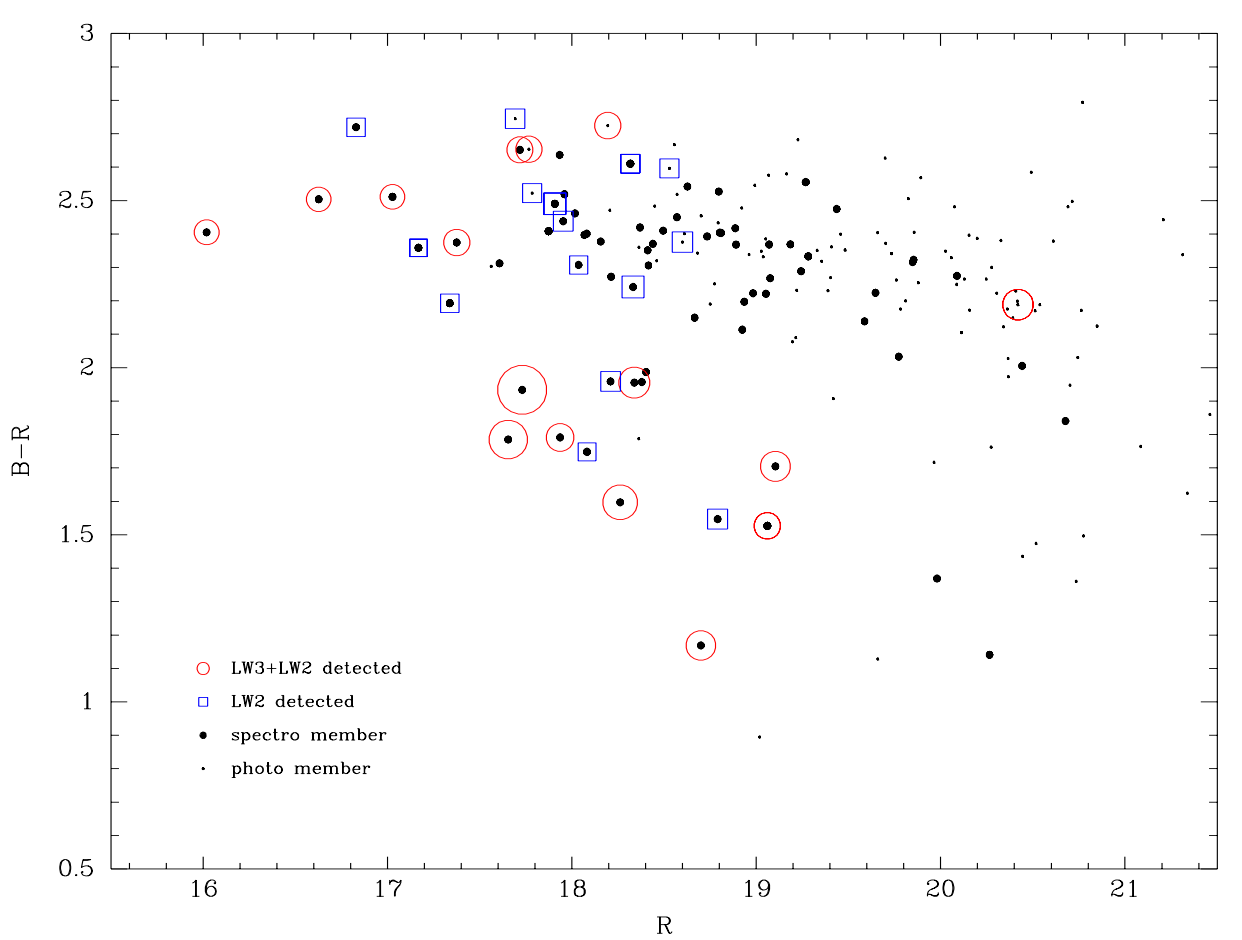

Fig. 7. Color-magnitude diagram towards Abell 1689. Galaxies whose cluster membership has been assessed from their photometric redshift are shown with the small black point. Spectroscopically confirmed members are shown with the large black points. Galaxies detected at $6.75(L W 2)$ and $15 \mu \mathrm{m}(L W 3)$ are indicated by resp. the (blue) squares and the (red) circles. The size of the latter symbols are proportional to the ISOCAM flux color $L W 3 / L W 2$. Note that almost all $L W 3$ sources were also detected in the $L W 2$ filter. For $L W 2$-only detected sources, an upper limit for the $L W 3$ flux was used to compute the MIR color. Only galaxies in the ISOCAM field of view are shown.

galaxies are known to be more frequent in ISO selected samples than in optical samples (Flores et al. 1999). It is also well known that the most extreme far-infrared emitters - the ULIRGs - are all mergers (Sanders \& Mirabel 1996). However, as we will show in Sect. 4.1, none of the MIR sources in Abell 1689 have far IR luminosities as high as ULIRGs.

The galaxy at the core of the X-ray cluster emission, ISO\#14, which is detected at $15 \mu \mathrm{m}$, has an intriguing morphology: its isophotes are ellipticals but its central surface brightness is magnitudes below that of elliptical or cD galaxies.

\subsubsection{Spectral class}

The dominant population among sources with a low $L W 3 / L W 2$ flux ratio are passive, $\mathrm{k}$-type, galaxies while $50-70 \%$ of the $L W 3$-bright sources are emission-line galaxies (see Fig. 4). The only AGN in our MOS sample (ISO\#41) is detected in both ISOCAM bands, with a $L W 3 / L W 2$ flux of 4 which is not unusual for an AGNdominated galaxy (Laurent et al. 2000).

One spectral class of particular interest for MIR selected samples is the so-called "e(a)" class: objects having moderate $[\mathrm{OII}]$ emission line and a strong $\mathrm{H}_{\delta}$ absorption line. Objects with such a spectral signature in the optical have been interpreted by Poggianti et al. (1999) as dusty starbursts. Observationally, e(a) is the typical spectral class of very luminous infrared galaxies (Poggianti \& Wu 2000) which are notoriously powered by dust enshrouded starbursts. In this survey, only one galaxy has an e(a) spectrum according to the strict $\mathrm{H} \delta$ threshold as defined by Dressler et al. (1999). However, other three spectra display similar characteristics, with weak to moderate [OII] emission and unusually strong early Balmer lines in absorption. The average rest-frame $<\mathrm{H} \theta+\mathrm{H} \eta+\mathrm{H} \zeta>/ 3$ of these galaxies is always higher than $5 \AA$ (see Table 5) and is much stronger than in the spectra of all the other spectral classes, except the $\mathrm{k}+\mathrm{a}$ 's. In these galaxies the $\mathrm{H} \delta$ line might be slightly more filled by emission than in the "proper" e(a) galaxies, but given the strong $<\mathrm{H} \theta+\mathrm{H} \eta+\mathrm{H} \zeta>/ 3$ their spectrum is likely to be the result of star formation and dust properties similar to those in e(a)'s, and we decided to include them in the e(a) class hereafter. e(a) spectra represent about $25 \%$ of our $L W 3$ sample, whereas all e(a)'s in the spectroscopic sample are $L W 3$ sources. Once again, this seems to confirm the existence of a connection between dusty star-forming galaxies and optical spectra with strong early Balmer lines and weak emission. The star formation rates and dust extinction of the e(a) galaxies will be presented in Sect. 4.1.3, where it will be shown that the SF activity in e(a)'s is the highest of all spectral classes.

Surprisingly, five $L W 3$ sources do not show any sign of current or recent star formation activity in their optical spectra. These galaxies have the lowest $L W 3$ fluxes 


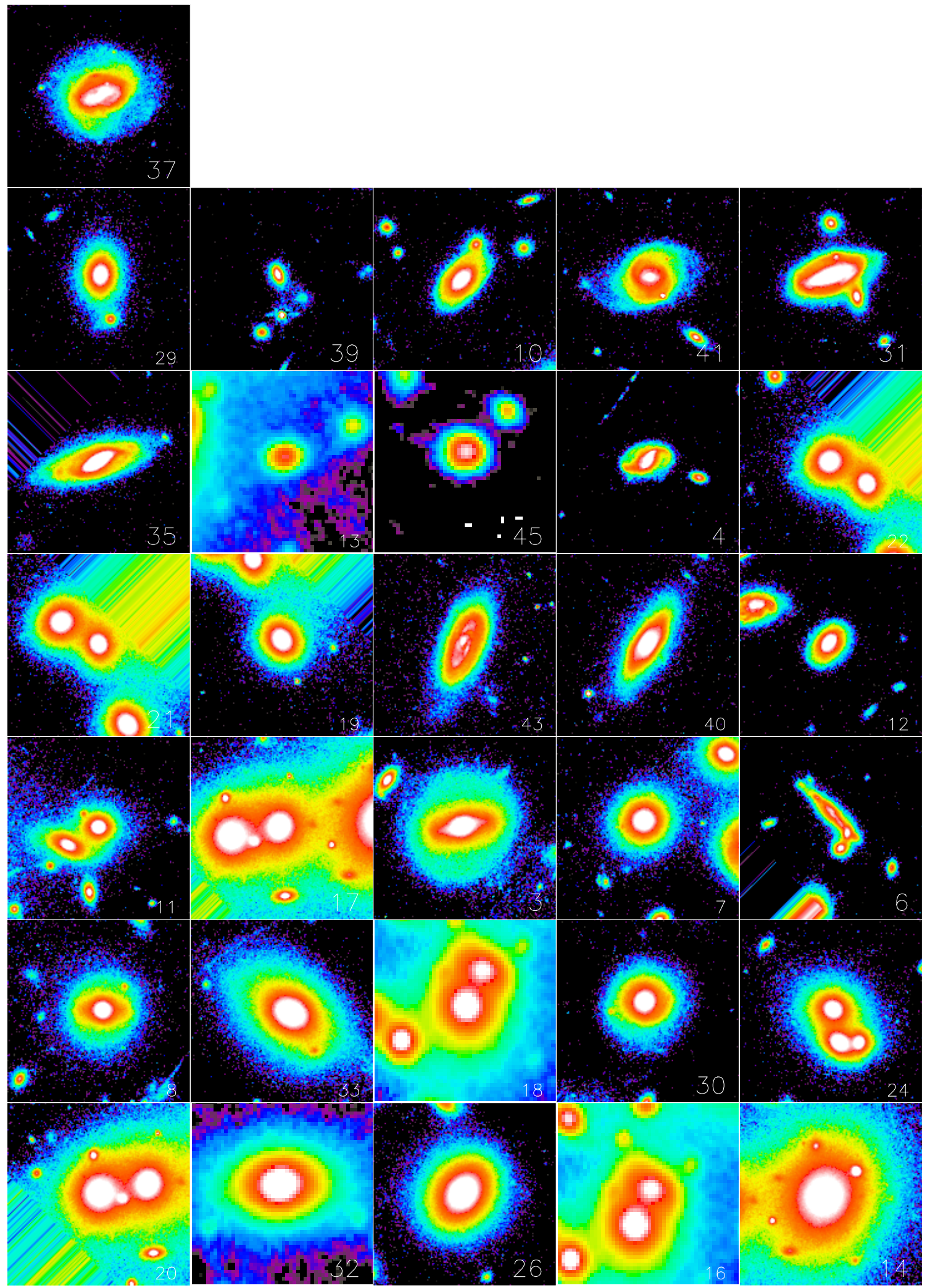

Fig. 8. HST/WFPC2 images of ISOCAM detected cluster members. They are ordered according to the increasing $L W 3 / L W 2$ flux ratio (from the lower left to the upper right panels). The spatial and intensity (logarithmic) scales are the same in all panels. For ISO\#32 and ISO\#45 which lie outside the HST survey field of view, NTT images are shown instead. Galaxies are labeled with the ISO ID number. Labels with a low-size text font indicate objects only detected at $6.75 \mu \mathrm{m}$. For these, an upper limit for the $L W 3$ flux was used to compute the MIR color. 
in the sub-sample and the origin of their LW3 emission will be discussed in Sect. 4.1.2. In total, less than $15 \%$ of the passive (k-type) galaxies and no post-starburst $(\mathrm{k}+\mathrm{a})$ galaxies are detected at $15 \mu \mathrm{m}$.

\subsubsection{The IR side of the $\mathrm{BO}$ effect}

The properties of the galaxies from our MIR samples can roughly be summarized as follows: sources with a low $L W 3 / L W 2$ flux ratio typically consist of luminous, red, passive early type galaxies, while those with a "red" MIR color are mainly luminous, blue, emission line, disturbed spiral galaxies - precisely the galaxies responsible for the optical Butcher-Oemler effects. Moreover, a small but significant fraction of $L W 3$ sources show no sign of current nor recent $\mathrm{SF}$ in their optical spectra.

\section{Discussion}

In the previous section, it has been shown that the majority of $L W 3$ sources have spectra with emission-lines indicative of ongoing star formation. Thus, qualitatively the optical SF estimators generally agree with the MIR data in identifying starforming galaxies, in about $70 \%$ of the cases. In this section, we will compare the $S F R$ derived from the [OII] line and from the MIR flux in a quantitative manner, with the purpose of evaluating how much of the SF activity remains undetected from the spectra.

\subsection{Total star formation rates in Abell 1689}

\subsubsection{Optical star formation rate}

Balmer hydrogen lines are commonly used to derive starformation rates in the optical. However for most of the cluster members, the $\mathrm{H}_{\alpha}$ line was outside our spectral domain. The $\mathrm{H}_{\beta}$ emission line is not reliable because it is polluted by the absorption line from A stars. Our spectral resolution is too low to properly subtract this contribution. Measurements in the UV, that are heavily affected by dust extinction as well, are not available. The only tracer at our disposal in the optical is the $[\mathrm{OII}]_{\lambda 3727}$ emission line. Several studies have emphasized the many problems of that line (e.g. Jansen et al. 2001; Charlot \& Longhetti 2001). Being in the blue, it is very sensitive to extinction. Moreover, its strength is not simply proportional to the number of ionizing photons; it depends very much on the metallicity/excitation of the ISM. As a result, the uncertainties in the $S F R$ derived from [OII] may be as high as a factor of 10 . Nevertheless, this line is used very often as a SF indicator, given the lack of alternatives in many studies.

$[\mathrm{OII}]_{\lambda 3727}$ could be measured in the spectra of 9 galaxies which are members of A1689 lying within the field of view of the ISOCAM survey. We have used the conversion of Kennicutt (1998):

$\operatorname{SFR}([\mathrm{OII}])=1.4 \times 10^{-41} L\left([\mathrm{OII}] / \mathrm{erg} \mathrm{s}^{-1}\right) M_{\odot} \mathrm{yr}^{-1}$ which is valid for solar abundance and a Salpeter IMF. The $S F R$ derived in A1689 galaxies ranges between $0.05 M_{\odot} \mathrm{yr}^{-1}$ and $0.5 M_{\odot} \mathrm{yr}^{-1}$ with an average and median among [OII]-detected galaxies of $0.2 M_{\odot} \mathrm{yr}^{-1}$ per galaxy (see Table 6). These values are lower limits, given that no extinction correction was applied. Moreover, a fraction of the [OII] flux from the galaxies might have been missed by the slitlet (see Sect. 4.2.1). After correcting for a canonical extinction in the optical of $1 \mathrm{mag}$ at $\mathrm{H}_{\alpha}$ (Kennicutt 1992), the average $S F R$ per star-forming galaxy rises to $0.5 M_{\odot} \mathrm{yr}^{-1}$. The highest optical $S F R$ measured in our sample, $1.15 M_{\odot} \mathrm{yr}^{-1}$, appears rather modest with respect to that found in other clusters at similar redshifts (e.g. AC 114 at $z=0.32$ Couch et al. 2001) and to the average $S F R$ measured in the coeval field (e.g. Tresse \& Maddox 1998) with slit spectroscopy.

\subsubsection{Mid infrared emission and star formation activity}

The MIR emission, despite its complexity, is arguably a reliable tracer of the star formation activity. The many different components at the origin of the emission in the ISOCAM bands have been discussed in detail in many papers (e.g. Genzel \& Cesarsky (2000), and references therein). In short, the main contributors are (a) unidentified infrared bands (UIBs) from photodissociation regions (PDR), (b) continuum emission by warm small grains heated by young stars or an AGN (c) continuum emission from the photosphere of evolved stars, and (d) emission lines from the ionized interstellar gas. The exact contribution of each component is difficult to be assessed using broad-band photometric data only. When no spectrum is available, the $L W 3 / L W 2$ color index provides a rough indication of the relative importance in the MIR regime of the starburst activity versus more quiescent spiral-like star formation, AGN activity and stellar emission, in the sense that a higher $L W 3 / L W 2$ generally indicates a more prevalent starburst activity (see the empirical models of Laurent et al. 2000). Of course, this single diagnostic is at some level degenerate and some a priori assumption on the origin of the MIR emission should be introduced.

In our sample, nuclear activity does not contribute much. Only one AGN, of Seyfert 1 type, is present in our optical spectroscopic database (see Sect. 3.2.3). Moreover, it is unlikely that Abell 1689 harbors obscured AGNs similar to those discovered in the most extreme ULIRGs (Sanders et al. 1988). The contribution of the nuclear activity seems to become prominent only in galaxies with a total infrared luminosity that exceeds $10^{12.3} L_{\odot}$ (Veilleux et al. 1999; Tran et al. 2001) while, as we will see in the following, all the galaxies in our sample have IR luminosities more than an order of magnitude lower.

A correlation between the $L W 3 / L W 2$ flux ratio and the $L W 3$ luminosity, shown in Fig. 9, is observed for the A1689 cluster members, especially when one takes into account the upper limits. This trend can be qualitatively understood considering that the $L W 3$ luminosity is an 
Table 6. Star formation rates of star-forming galaxies in A1689.

\begin{tabular}{lccccc}
\hline $\begin{array}{l}\mathrm{ID}^{a} \\
(\mathrm{MOS})\end{array}$ & $\begin{array}{c}\mathrm{ID}^{b} \\
(\mathrm{ISO})\end{array}$ & $\begin{array}{c}F_{15} / F_{K^{\prime}}{ }^{c} \\
-\end{array}$ & $\begin{array}{c}L_{\mathrm{IR}}{ }^{d} \\
10^{10} L_{\odot}\end{array}$ & $\begin{array}{c}S F R(\mathrm{IR})^{e} \\
M_{\odot} \mathrm{yr}^{-1}\end{array}$ & $\begin{array}{c}S F R([\mathrm{OII}])^{f} \\
M_{\odot} \mathrm{yr}^{-1}\end{array}$ \\
\hline 2 & - &.. & $<0.81$ & $<1.4$ & $0.46 \pm 0.02$ \\
7 & 3 & $0.5 \pm 0.2$ & 1.32 & $2.2 \pm 0.9$ & $0.06 \pm 0.03$ \\
14 & 4 & $1.4 \pm 1.0$ & 0.93 & $1.6 \pm 1.2$ & $0.18 \pm 0.03$ \\
16 & 6 &.. & $0.81:$ & $1.4:$ & $0.23 \pm 0.01$ \\
23 & 10 & $1.6 \pm 0.5$ & 1.79 & $3.0 \pm 1.0$ & $0.30 \pm 0.02$ \\
29 & 14 & $0.1 \pm 0.1$ & 1.35 & $2.3 \pm 0.9$ & $<0.05$ \\
.. & 17 & $0.3 \pm 0.1$ & 1.26 & $2.1 \pm 1.0$ &.. \\
. & 21 & $0.5 \pm 0.4$ & 0.96 & $1.6 \pm 1.2$ &.. \\
43 & 26 & $0.2:$ & $0.81:$ & $1.4:$ & $<0.05$ \\
46 & 30 & $0.2:$ & $0.81:$ & $1.4:$ & $<0.05$ \\
48 & - &.. & $<0.81$ & $<1.4$ & $0.15 \pm 0.02$ \\
49 & 31 & $0.9 \pm 0.3$ & 1.79 & $3.0 \pm 1.0$ & $0.24 \pm 0.03$ \\
51 & 32 & $0.1:$ & $0.81:$ & $1.4:$ & $<0.05$ \\
54 & 35 & $0.8:$ & $1.17:$ & $2.0:$ & $0.19 \pm 0.02$ \\
55 & 37 & $2.9 \pm 0.4$ & 6.19 & $10.5 \pm 1.7$ & $0.10 \pm 0.03$ \\
.. & 39 & $0.9 \pm 0.5$ & 1.08 & $1.8 \pm 1.1$ &.. \\
61 & 41 & $2.7 \pm 0.6$ & 2.38 & $4.0 \pm 0.9$ & $<0.05$ \\
69 & 43 & $<0.7$ & $<0.81$ & $<1.4$ &.. \\
72 & 45 & $6.2 \pm 1.1$ & 3.78 & $6.4 \pm 1.2$ &.. \\
\hline
\end{tabular}

Notes: this table includes "active" cluster members in the ISOCAM field of view that either have emission lines in their optical spectra or that are detected at $15 \mu \mathrm{m}$. ${ }^{a}$ ".." indicates that no MOS spectra is available. ${ }^{b}$ "_" indicates that the galaxy has not been detected by ISOCAM. ${ }^{c} 15 \mu \mathrm{m}$ to $2.2 \mu \mathrm{m}$ flux ratio. $K^{\prime}$ magnitudes were taken from de Propris et al. (1999). ${ }^{d}$ Total IR luminosity estimated from the $15 \mu \mathrm{m}$ luminosity. We used the following formulae: $\log \left(\nu L_{\nu}[12 \mu \mathrm{m}]=0.494+0.955 *\right.$ $\log \left(4 \pi D^{2} \nu F_{\nu}[15 \mu \mathrm{m}]\right)$ (conversion to the equivalent rest-frame "IRAS" luminosity at $\left.12 \mu \mathrm{m}\right) L_{\mathrm{ir}}=0.89 *\left(\nu L_{\nu}[12 \mu \mathrm{m}]\right)^{1.094} L_{\odot}$ (calibration of Chary \& Elbaz 2001). The luminosities indicated with ":" have large errors and may be upper limits (see Paper I). ${ }^{e}$ Infrared star formation rate derived from $L_{\mathrm{IR}}$. For galaxies with $F_{15} / F_{K^{\prime}}$ below 0.1 , the photospheric emission becomes greater than $50 \%$, and the estimated $S F R$ is overestimated by a factor of 2 . Above 0.5 , the correction is negligible. The AGN activity in MOS ID\#61 might contribute to the MIR luminosity, and hence its derived $S F R$ might be overestimated.

${ }^{f}$ Star formation rate estimated from the [OII] luminosity, not corrected for any optical extinction or slit aperture correction.

indicator of the star formation activity ${ }^{7}$. Passive galaxies have a mid-infrared spectrum in which the Rayleigh-Jeans tail of the dominant old stellar component prevails; for a pure stellar contribution approximated by a $T=3500 \mathrm{~K}$ black body redshifted to $z=0.2$, the MIR color index is as low as $\log L W 3 / L W 2=-0.6$. The MIR spectrum of galaxies with a low to moderate star formation rate, typical of normal spirals, is dominated by the UIBs; their MIR color index is typically $\log L W 3 / L W 2=0$ to 0.5 at $z=0.2$ (see Fig. 11 in Laurent et al. 2000). Finally, the MIR spectrum of galaxies with a high $S F R$, i.e. starbursts, is dominated for $\lambda>10 \mu \mathrm{m}$ by continuum emission from hot dust; their $L W 3 / L W 2$ ratio increases to values as high as $\log L W 3 / L W 2=1$. The correlation between the MIR color index and the $15 \mu \mathrm{m}$ flux is kept when the latter is normalized by the near-infrared $K^{\prime}$ flux at $2.2 \mu \mathrm{m}$, a tracer of the total stellar luminosity (see Fig. 10). Pure stellar MIR emitters have $\log L W 3 / K^{\prime}=-1.1$ (after $k$-correction) while in dusty active galaxies this ratio may reach $\log L W 3 / K^{\prime}=-0.5$. The diagrams presented

$\overline{7}$ In contrast, the $L W 2$ flux observed at the redshift of Abell 1689 (rest-frame $5.7 \mu \mathrm{m}$ ) is mostly due to stellar emission and is hence a worse tracer of the star formation activity. Indeed, the $L W 3 / L W 2$ ratio and the $L W 2$ flux are not correlated. in Figs. 9 and 10 show that all $L W 3$-detected galaxies in A1689 have a $15 \mu \mathrm{m}$ flux which is much higher than expected for a Rayleigh-Jeans emission. The contribution from photospheric stellar emission may be up to $50 \%$ for galaxies with the lowest $L W 3 / K^{\prime}$ flux ratio, on average is about $20 \%$, and less than $2 \%$ for the $15 \mu \mathrm{m}$-bright galaxies.

Therefore the $15 \mu \mathrm{m}$-detected population is composed of star-forming cluster members with some (5 to 7) galaxies with $L W 3 / L W 2$ ratios in the range typical for normal spirals, while the remaining (3 to 5 ) galaxies have higher $L W 3 / L W 2$ ratios suggestive of a current starburst.

\subsubsection{Infrared star formation rate}

The level of star-formation activity may be estimated in a more quantitative but indirect way through the relation between the mid and far-infrared luminosities. Dust enshrouded young stars emit radiation which is reprocessed and emitted by dust mainly in the wavelength domain 40-1000 $\mu \mathrm{m}$. Hence the FIR luminosity is one of the best tracers of the global $S F R$ (Kennicutt 1998). In a sample of nearby IRAS galaxies observed with ISOCAM, Chary \& Elbaz (2001) found strong correlations between the luminosity at 12 and $15 \mu \mathrm{m}$ and the total IR luminosity at 

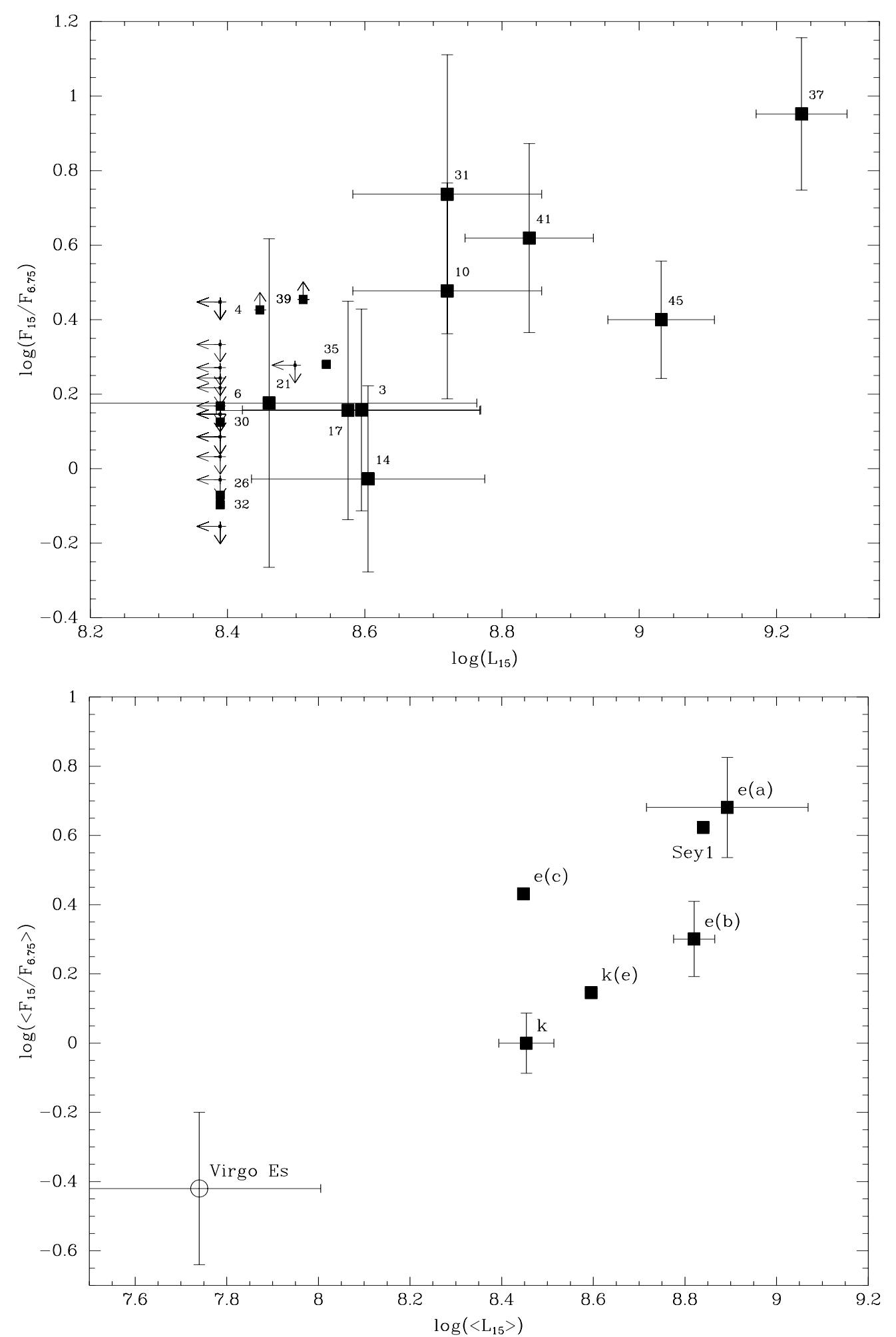

Fig. 9. Mid-infrared $L W 3 / L W 2$ color index versus $15 \mu \mathrm{m}$ luminosity (in $L_{\odot}$ ) for ISOCAM cluster members. Top: individual values. The ISO ID number is indicated for galaxies detected at both $6.75 \mu \mathrm{m}$ and $15 \mu \mathrm{m}$. Bottom: averaged values per spectral class. The errors are the deviation from the mean. Only galaxies detected in both ISOCAM bands were used. The open circle corresponds to the average values of early-type galaxies in the Virgo cluster (Boselli et al. 1998). The $15 \mu \mathrm{m}$ luminosities were computed using the formula $L_{15}=4 \pi D^{2} F_{15} \delta_{\nu}$ where $\delta_{\nu}$ is the $L W 3$ filter width.

$\lambda 8-1000 \mu \mathrm{m}$. This result, which confirms the early works based on IRAS studies (e.g. Spinoglio et al. 1995), corroborates the idea that the MIR regime is a good tracer of the star formation rate in galaxies. At the redshift of Abell 1689, the central wavelength of the $L W 3$ filter corresponds to rest-frame $\lambda=12.7 \mu \mathrm{m}$, which is close to 

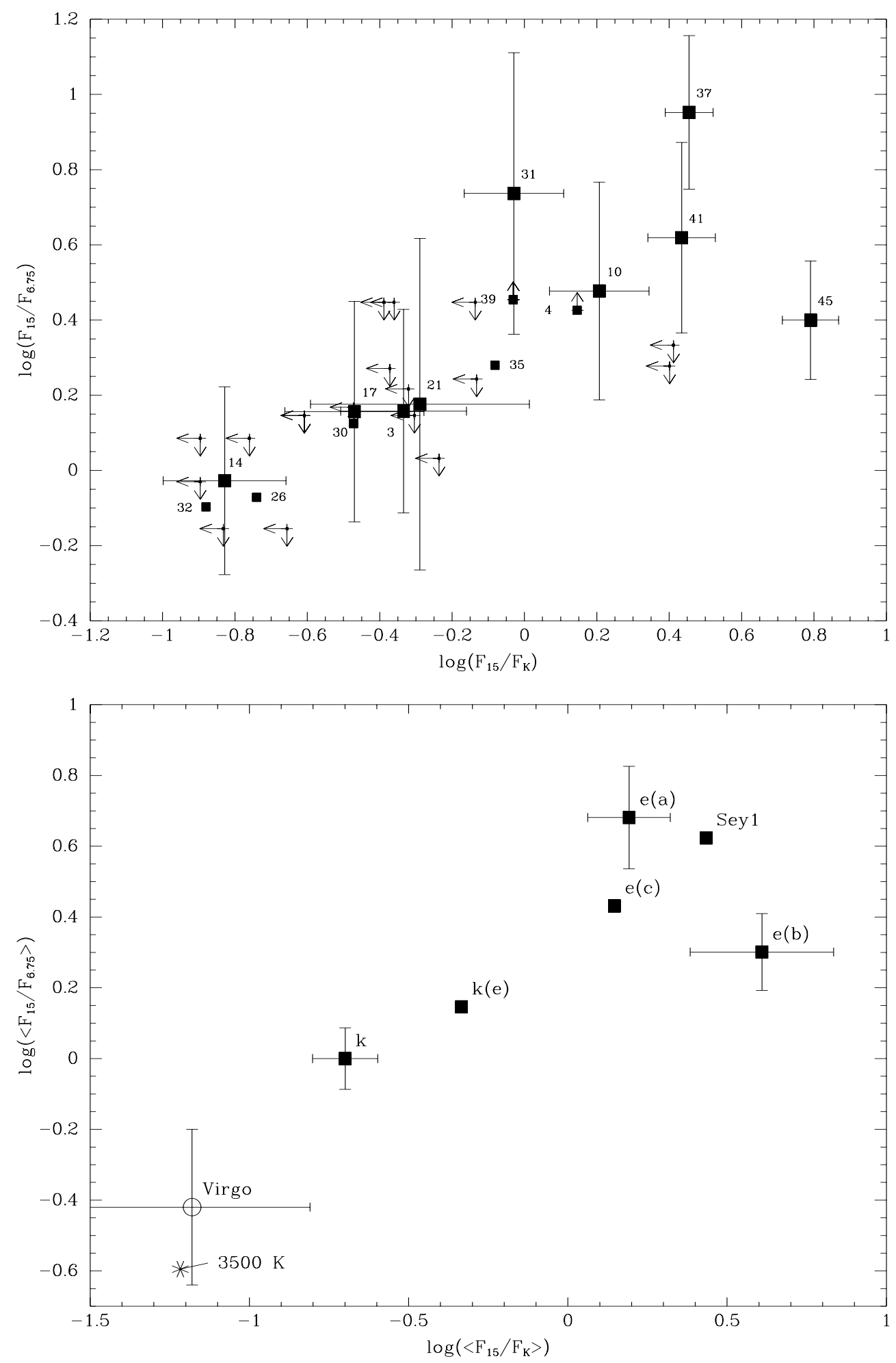

Fig. 10. Mid-infrared $L W 3 / L W 2$ color index versus $L W 3 / K^{\prime}$ flux ratio for ISOCAM cluster members. Top: individual values. The ISO ID number is indicated for galaxies detected at both $6.75 \mu \mathrm{m}$ and $15 \mu \mathrm{m}$. Bottom: averaged values per spectral class. The errors are the deviation from the mean. Only galaxies detected in both ISOCAM bands were used. The star indicates the position of a $3500 \mathrm{~K}$ blackbody which approximates the SED of ellipticals. The associated arrow shows the $k$-correction to apply at $z=0.18$. The open circle corresponds to the average values of early-type galaxies in the Virgo cluster (Boselli et al. 1998).

the central wavelength of the IRAS $12 \mu \mathrm{m}$ band. We converted the $L W 3$ fluxes into IRAS equivalent $12 \mu \mathrm{m}$ fluxes, taking into account the differences in the bandwidth and response function between the two filters. Adopting the 
MIR-FIR relations derived by Chary \& Elbaz (2001), we estimated the far-infrared luminosity for all $L W 3$ sources in A1689. For LW3-detected galaxies, we find that the infrared luminosity ranges between $0.9 \times 10^{10} L_{\odot}$ and $6.2 \times 10^{10} L_{\odot}^{8}$. Therefore, none of the MIR sources in A1689 reaches the luminosities of Luminous Infrared Galaxies. The average infrared luminosity is $2 \times 10^{10} L_{\odot}$.

We computed the star formation rate from the IR luminosity following the conversion of Kennicutt (1998):

$S F R(\mathrm{IR})=1.7 \times 10^{-10}\left(L_{\mathrm{ir}} / L_{\odot}\right) M_{\odot} \mathrm{yr}^{-1}$.

The assumptions are continuous bursts lasting 10-100 Myr and the same Salpeter IMF as that adopted when deriving $S F R([\mathrm{OII}])$. With this conversion, given the sensitivity of our ISOCAM survey, galaxies with a $S F R$ lower than $1.4 M_{\odot} \mathrm{yr}^{-1}$ would not have been detected.

The $S F R(\mathrm{IR}) \mathrm{s}$ derived in this way are listed in Table 6 . In Abell 1689, the $L W 3$ sources with accurate $L W 3$ flux (11 in 16) have a $S F R(I R)$ that ranges between 1.6 and $11 M_{\odot} \mathrm{yr}^{-1}$ with an average of $3.5 M_{\odot} \mathrm{yr}^{-1}$ per galaxy and a median value of $2.3 M_{\odot} \mathrm{yr}^{-1}$.

Deep VLA radio maps of Abell 1689 will soon become available (Morrison 2000). The radio continuum emission provides another excellent tool to probe the total star formation rate in galaxies, therefore it will be interesting to compare these new data with our MIR-based estimate of the star formation rate.

Comparisons with the coeval field are still difficult due to the lack of data. The situation will remain as such until the ELAIS ISOCAM surveys are fully released (Oliver et al. 2000). For the time being, $15 \mu \mathrm{m}$ data have been published for a couple of small fields: the CFRS field 1415+52 (Flores et al. 1999) and the Hubble Deep field (Serjeant et al. 1997; Aussel et al. 1999) which includes galaxies at an average redshift of $z=0.8$. At this distance, given their completeness limits $(0.25 \mathrm{mJy}$ for the CFRS field and $0.10 \mathrm{mJy}$ for the HDF), these surveys picked up mostly luminous infrared galaxies $\left(L_{\mathrm{IR}}>10^{11} L_{\odot}\right)$ with star formation rates much higher than in Abell 1689. The ISOCAM survey of the whole CFRS fields provided a sample of 5 galaxies with a redshift between 0.1 and 0.3 and a $15 \mu \mathrm{m}$ flux higher than the sensitivity limit of the A1689 survey (Flores et al., in preparation). Their median $S F R$ inferred from their mid-infrared flux with the same method as in this paper, $2.2 M_{\odot} \mathrm{yr}^{-1}$, is similar to the median SFR measured in the star-forming galaxies of Abell 1689. A comparison per morphological type, which would be more fair, is yet impossible due to the size of these samples.

\footnotetext{
${ }^{8}$ Note that below infrared luminosities of $10^{10} L_{\odot}$, the contamination by cirrus might be important. In this sample, it is negligible.
}

\subsection{Hidden star formation in Abell 1689}

We will now compare the star formation rates derived in the infrared and in the optical for the $L W 3$-detected galaxies.

\subsubsection{Star formation missed in the optical}

As shown in Fig. 11, the ratio $S F R(\mathrm{IR}) / S F R([\mathrm{OII}])$ ranges between about 10 and 100 with a median value of 12 (a mean of 30) when only considering emissionline galaxies for which the [OII] flux could be measured and for which the $15 \mu \mathrm{m}$ flux is well determined (5 galaxies in total). Adding the $L W 3$ sources with undetected $[\mathrm{OII}]_{\lambda 3727}$ line and assigning to its flux a conservative upper limit of $5 \times 10^{-17} \mathrm{erg} \mathrm{cm}^{-2} \mathrm{~s}^{-1}$, the median $S F R(\mathrm{IR}) / S F R([\mathrm{OII}])$ raises to 30 . Therefore, one misses at least $90 \%$ of the star formation activity when estimating the latter from the $[\mathrm{OII}]$ optical line.

Note that a high $S F R(\mathrm{IR}) / S F R([\mathrm{OII}])$ is more likely the result of a high extinction rather than a strong underestimate of the [OII] flux caused by slit aperture effects. Surely, excentered H II regions might have been missed by the slitlets which were centered on the nucleus and had a random orientation with respect to the galaxy position angle. We tried to quantify aperture losses extrapolating the surface brightness profile of the [OII] line along our $1.3^{\prime \prime}$-wide slits to the entire galaxy. We obtained upper limits for the aperture correction factor ranging between 1.2 and 1.9 with an average of 1.6. Therefore, even after applying this correction, $S F R(\mathrm{IR})$ and $S F R([\mathrm{OII}])$ cannot be reconciled without a strong extinction. We remind the reader that the "standard" dust correction generally adopted for spirals ( $1 \mathrm{mag}$ at $\mathrm{H} \alpha$ ) is equivalent, given the $S F R$ calibrations of Kennicutt (1992), to assuming $S F R(\mathrm{IR}) / S F R(\mathrm{OII})=2.5$. Undoubtedly, an important fraction of the star formation activity in Abell 1689 is dust enshrouded. Using deep radio centimetric observations to trace star formation in the $z=0.41$ cluster CL0939+4713, Smail et al. (1999) reached a similar conclusion.

The level of dust enshrouded star formation depends however very much on the galaxy spectral type, as can be seen in Figs. $9 \mathrm{~b}$ and $10 \mathrm{~b}$ where the average $L W 3$ luminosity, $L W 3 / K^{\prime}$ and $L W 3 / L W 2$ flux ratios are plotted for each spectral class.

In Sect. 3.3.4, it has been reported that all e(a) galaxies in our sample are $L W 3$ sources and that about $25 \%$ of the $L W 3$ sources have e(a) spectra. We see from Fig. 9b that galaxies with an e(a) signature have the largest $L W 3$ luminosity and $L W 3 / L W 2$ flux ratio, i.e. their MIR emission is clearly powered by a starburst rather than quiescent star-formation. In the most extreme galaxy among these dusty starbursts, ISO\#37, the total $S F R$ is underestimated by a factor of 100 when computed from the [OII] line (see Fig. 11). We note however that, whereas e(a)'s are common among the $L W 3$ subsample and have rather 


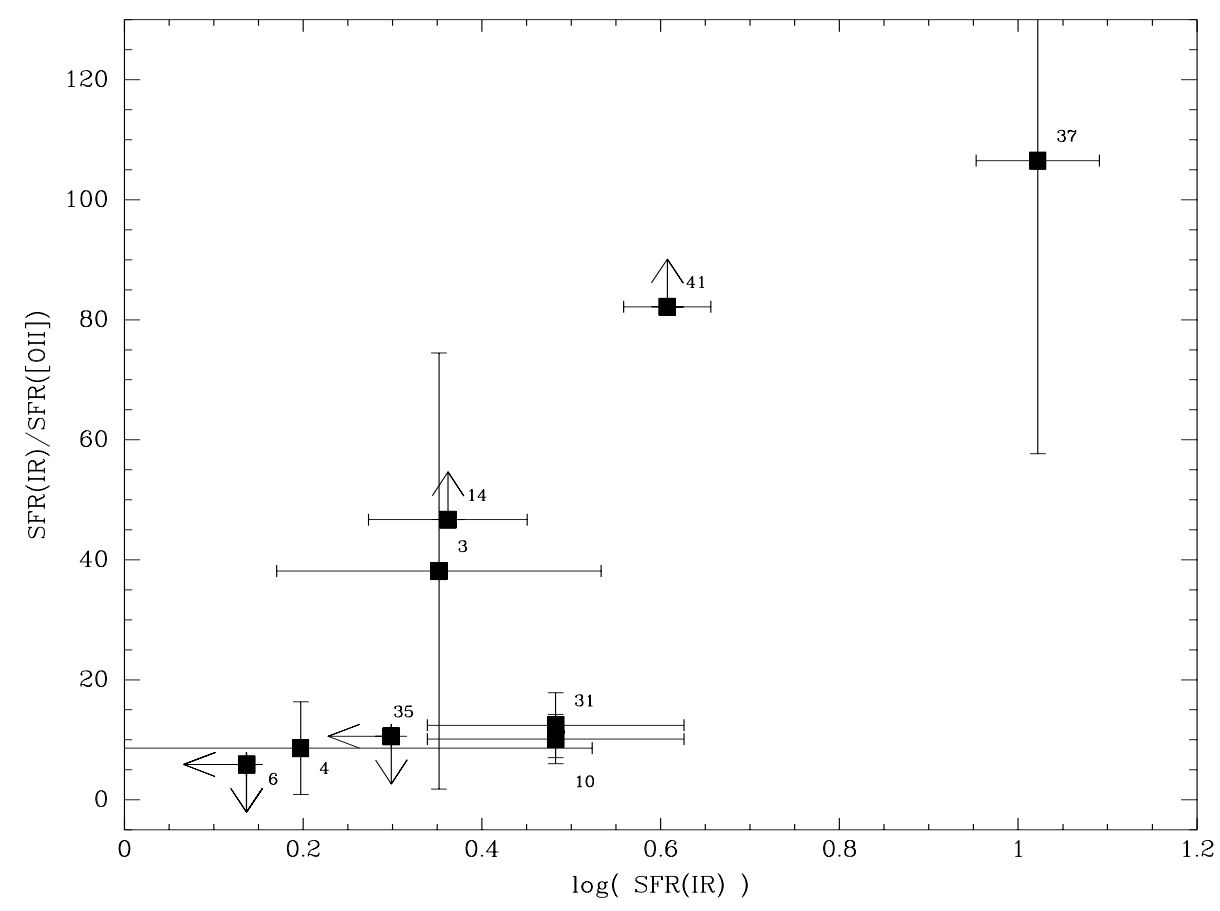

Fig. 11. Ratio between the star formation rate derived from the infrared to the star formation estimated from the flux of the $[\mathrm{OII}]_{\lambda 3727}$ line versus $\log S F R(\mathrm{IR})\left(\right.$ in $M_{\odot} \mathrm{yr}^{-1}$ ). No extinction or aperture corrections have been applied to $S F R([\mathrm{OII}])$. The ISO ID number is indicated.

large $S F R$ s, they constitute in number only $5 \%$ of the total cluster population.

It was suggested that some of the numerous $\mathrm{k}+\mathrm{a}$ galaxies could be extreme e(a)'s in which the [OII] line is totally extinguished (Smail et al. 1999). However, no k+a is detected in the mid-infrared regime in Abell 1689. Galaxies of this spectral class do not host hidden star formation activity up to the ISOCAM sensitivity limit of $1.4 M_{\odot} \mathrm{yr}^{-1}$. They are rather likely to be genuine "poststarburst" galaxies or at least galaxies where star formation has stopped.

It has been noted in Sect. 3.3.4 that some of the $L W 3$ sources do not show signs of SF activity in their spectra. In fact, at least five galaxies ${ }^{9}$, namely ISO\#3, $14,26,30,32$ that are considered as "passive" in view of their optical properties - a k-/k(e)-type spectrum and, sometimes, an early-type morphology (Es, S0s) - are detected in the $15 \mu \mathrm{m}$ band. The emission at the same restframe wavelength from ellipticals in Virgo and Coma was interpreted as dominated by photospheric emission from old stars (Boselli et al. 1998). However, stellar emission is not likely to be the dominant source of the 15 micron flux observed in the optically-passive galaxies of A1689, as judged from their $L W 3 / L W 2$ and $L W 3 / K^{\prime}$ flux ratios (discussed in Sect. 4.1.2). They are much higher than those of Virgo's ellipticals while the $15 \mu \mathrm{m}$ luminosity increases by a factor of 5 (see Figs. 9b and 10b). Placed at the redshift of Abell 1689, the nearby ellipticals from

\footnotetext{
${ }^{9}$ Out of a total of $16 L W 3$ sources (30\%). The maximum percentage is $50 \%$ taking into account the three $L W 3$ sources absent in our MOS sample.
}

the sample of Boselli et al. (1998) would have $L W 3$ fluxes below $0.1 \mathrm{mJy}$; therefore none of them would have been detected by our survey. If part of the $L W 3$ emission of the "passive" A1689 galaxies is really, as we believe, due to star formation, the inferred value of the $S F R$ is modest but significant: up to $2 M_{\odot} \mathrm{yr}^{-1}\left(1 M_{\odot} \mathrm{yr}^{-1}\right.$ applying a correction factor due the photospheric emission) and 2040 times higher than that derived in the optical from the upper limit of the [OII] line (see Fig. 11).

\subsubsection{Star formation missed in the infrared}

Conversely, one could ask whether all star formation shows up in the mid-infrared. In principle, in environments with small amounts of dust, the UV photons emitted in SF regions are not re-processed and are missed in the MIR. In our sample, one galaxy with strong equivalent widths of the optical emission lines was not detected at $15 \mu \mathrm{m}$ (MOS\#48). It turns out however that it has an $R$-band magnitude below 19.5. No galaxies have been detected in $L W 3$ in this luminosity domain, apart from one object with $R=20.4$ (ISO\#39) for which the cluster membership is uncertain as it is based on its photometric redshift. The "optical" SFR measured for galaxy MOS\#48 is only $0.15 M_{\odot} \mathrm{yr}^{-1}$. The non-detection is then likely due to the lack of sensitivity of ISOCAM for low mass galaxies with integrated $S F R$ below $1.4 M_{\odot} \mathrm{yr}^{-1}$. Therefore, this galaxy would not have been detected even if the infrared to optical $S F R$ was a factor of 10 . Sensitivity problems on the MIR side may also explain why two e(c) galaxies in the ISOCAM field of view were not detected as well (MOS\#2 and MOS\#69). 
Given the low-level of star formation undetected by ISOCAM in faint blue galaxies and the paucity of this kind of objects even in the photometric sample (see the colormagnitude diagram in Fig. 7), the MIR emission should provide a good rough estimate of the total SFR in the cluster. Of course, our optical and infrared surveys missed the faint end population of star-forming dwarf galaxies. If their luminosity function is steep enough, one might underestimate significantly the total star formation rate in the cluster.

\subsubsection{Dust extinction and the cluster environment}

The high $15 \mu \mathrm{m}$ luminosities, $L W 3 / K^{\prime}$ color index and $S F R(\mathrm{IR}) / S F R$ (opt) ratios measured in A1689 clearly indicate that the star-forming galaxies in the cluster are affected by strong dust obscuration. Does this high level of extinction result from the cluster environment? Such an effect would not be unexpected. Ram-pressure and galactic harassment contribute to strip the outer gas reservoir of gas-rich galaxies falling in the cluster environment. The star-formation activity is then quenched in the disk while it is maintained longer in the central regions which are less under the influence of the outside environment but are more affected by internal extinction. The increasing contribution of the dust-obscured nuclear or circumnuclear SF would be at the origin of a correlation between the apparent extinction and the Hi deficiency. Indeed, studying a sample of nearby spiral galaxies mapped by ISOCAM, Roussel et al. (2001) found that the ratio of the MIR to optical diameter decreases when the H I deficiency increases while the mean FIR to $\mathrm{H}_{\alpha}$ flux ratio rises along with central concentration of the MIR emission and hence of the warm dust. Besides, according to Moss \& Whittle (2000), circumnuclear star-formation is more common in rich clusters than in the field.

On the other side, dust enshrouded star formation episodes are commonly found in field luminous infrared galaxies (Sanders \& Mirabel 1996) - a lot of them being however interacting systems -, and recent studies indicate that most of the star-formation activity could be hidden even in UV-bright low-mass galaxies, such as the blue compact dwarf galaxies (Hunt et al. 2001). It is then not unlikely that SF in some, if not the majority, of the galaxies that fell in the cluster was already dust obscured. Understanding whether dust extinction is enhanced in galaxies as a consequence of the cluster environment will be possible only when MIR field surveys will have provided sufficient data for a statistical comparison with clusters such as A1689.

\section{Summary and conclusions}

We have presented the follow-up study of a mid-infrared ISOCAM survey of the $z=0.18$ rich cluster of galaxies Abell 1689 (Paper I). We compiled optical imaging, photometric and spectroscopic data for about 100 galaxies lying within the inner $\mathrm{Mpc}^{2}$ of the cluster. This database was used to assess their cluster membership likelihood and characterize their morphological type and spectral class. The spectroscopic sample includes $75 \%$ of the galaxies brighter than $R=17.75$ with a photometric redshift consistent with a cluster membership and $75 \%$ of the ISOCAM mid-infrared members. The optical properties of the optically selected cluster members seem to already differ from those of the galaxy populations in typical rich clusters in the nearby universe. In particular, we confirm the high fraction of blue galaxies previously reported in this cluster. Focussing on the properties of the mid-infrared emitters, we found that:

(1) The redshift histogram of ISOCAM galaxies towards A1689 shows a prominent excess of sources at the baricentric redshift of the cluster, $z=0.184$ as determined from our spectroscopic sample. They are undoubtly related to the cluster environment.

(2) The majority of the sources detected at $15 \mu \mathrm{m}$ are luminous, blue, emission-line, morphologically disturbed spiral galaxies, i.e. the star-forming galaxies usually associated with the "Butcher-Oemler" effect. Sources with the lowest $L W 3 / L W 2(15 \mu \mathrm{m}$ to $6.75 \mu \mathrm{m})$ flux ratio, in particular those undetected at $15 \mu \mathrm{m}$, typically consist of luminous early-type galaxies with passive spectra, i.e. with no signs of current or recent star formation.

(3) However, at least five $15 \mu \mathrm{m}$ sources $(\geq 30 \%$ of the $L W 3$-subsample) do not show any sign of star-formation activity in their optical spectrum.

(4) More than $70 \%$ of the emission-line galaxies in our spectroscopic sample are detected at $15 \mu \mathrm{m}$. The starforming dwarf galaxies were too faint to be detected by ISOCAM. All four cluster members with weak to moderate emission lines and unusually strong early Balmer lines in absorption ("e(a)" class), typical of dusty starbursts, are $L W 3$ sources. They have the highest $15 \mu \mathrm{m}$ to $6.75 \mu \mathrm{m}$ flux ratio in our sample. None of the galaxies with a post-starburst optical spectrum ("k+a" galaxies) have been detected at $15 \mu \mathrm{m}$.

The relative high values of the $L W 3 / L W 2$ and $L W 3 / K^{\prime}$ color indexes indicate that the $L W 3$ luminosity of the $15 \mu \mathrm{m}$ emitters is not dominated by stellar emission. The AGN activity is at low level in the cluster. The ISOCAM $L W 3$ emission is hence a reliable tracer of the dustobscured star formation activity. We have then estimated the total infrared luminosity and inferred star formation rate from the $15 \mu \mathrm{m}$ (12.7 $\mu \mathrm{m}$ rest-frame) luminosity and compared it with that derived in the optical from the flux of the [OII] line. We found that:

(1) There is no instance of Luminous Infrared Galaxies (LIGs) in Abell 1689. The highest total infrared luminosity, $6.2 \times 10^{10} L_{\odot}$, is measured in an e(a) galaxy with a derived star formation rate of about $11 M_{\odot} \mathrm{yr}^{-1}$. Its high $L W 3 / L W 2$ ratio is consistent with star formation occurring in a dust-enshrouded burst mode. SFRs up to $2 M_{\odot} \mathrm{yr}^{-1}$ are estimated in the apparently passive 
k-type galaxies. As seen from the MIR window, these objects form stars in a mode typical of spirals. On the other hand, the upper limit of the total $S F R$ for k+a galaxies, 1.4 $M_{\odot} \mathrm{yr}^{-1}$, is consistent with the absence of a strong SF activity in these objects.

(2) The median $S F R(I R)$ of the $L W 3$-detected galaxies is $2 M_{\odot} \mathrm{yr}^{-1}$ while the median $S F R(\mathrm{opt})$ of the [OII]-detected galaxies is only $0.2 \quad M_{\odot} \mathrm{yr}^{-1}$. For galaxies in common to both samples, the ratio $S F R(\mathrm{IR}) / S F R(\mathrm{opt})$ is very high and ranges between 10 and 100, being highest among e(a) galaxies. An underestimate of the [OII] luminosity and hence of $S F R$ (opt) due to slit effects alone cannot account for the differences between the optical and infrared indicators of the star formation rate.

We conclude that a significant portion of the star formation activity taking place in Abell 1689 is hidden in the cluster members when observed through the optical window. At least $90 \%$ of the SF is missed when estimated from the [OII] line, which is known anyway as an unreliable, although often used, tracer of the $S F R$.

Comparing the $S F R$ and the amount of dust extinction of A1689 members with those of coeval field galaxies would be useful to investigate in details the effects of the cluster environment on the star formation history of galaxies. It would however require to understand the complex dynamics of this rich cluster and, in particular, to locate the MIR sources in its different sub-units.

Acknowledgements. First of all, we wish to thank members of the MORPHS collaboration, in particular Warrick Couch and Ian Smail for providing us the reduced HST images and morphological info prior to publication. The input we got from Ian Smail on the original manuscript helped a lot improving it. We thank the referee, Dr. Jørgensen, for his useful comments. We are grateful to Chris Lidman, Adam Stanford, Roberto De Propris and Simon Dye for giving us their optical/nearinfrared images and photometric data towards Abell 1689 . Many thanks to the NTT team at La Silla for their precious help with the spectroscopic observations. PAD and BMP acknowledge support from the network Formation and Evolution of Galaxies set up by the European Commission under contract ERB FMRX CT96086 of its TMR program.

\section{Appendix A: Completeness of the MOS sample}

We used the sample of photometric members by Dye et al. (2001) to assess the completeness of our MOS survey. Dye et al.'s survey is complete up to $B=23.7$; unfortunately it does not totally cover our MOS field of view (see Fig. 2). We therefore restricted our analysis to the $5^{\prime} \times 6^{\prime}$ field of view where photometric redshifts are available.

First of all, we note that the photometric redshifts are reliable: the cluster membership of almost $90 \%$ of the photometric members with MOS data is confirmed with our direct redshift measurements and the average photometric and spectrophotometric redshifts differ by less than $10 \%$ (see Fig. 1).
As shown in Fig. A.1, we have obtained spectra for $75 \%$ of the cluster members brighter than $R=17.75$. For $R<19.5$, we sampled only about $40 \%$ of the photometrically confirmed cluster members while we missed most of the faintest dwarf galaxies with $R>20$. The histogram of the $B-R$ color index of photometric and spectroscopic cluster members is presented in Fig. A.2. As shown in the top panel, the comparison of these two distributions reveals that there is a weak color bias in our spectroscopic sample, which includes relatively more blue than red galaxies. Galaxies bluer than $B-R<1.75$ (that accounts for $15 \%$ of the spectroscopic sample) are overrepresented by $60 \%$. Note however that the photometric sample most likely misses the faintest bluest galaxies in the cluster because the photometric redshift technique does not work well for such deviant objects, and this acts to counterbalance the bias.

\section{Appendix B: Blue fraction in Abell 1689}

Our spectroscopic survey of Abell 1689 allows us to plot a color-magnitude diagram including only confirmed cluster members. One may then, in principle, estimate the blue fraction without the uncertainty introduced in photometric methods by the background correction. For a fair comparison with the latest studies on the subject, we computed $f_{\mathrm{B}}$ using the definition proposed by Margoniner et al. (2001,hereafter MdC01). We selected all galaxies inside a clustercentric radius of $0.7 \mathrm{Mpc}^{10}$ with an absolute red magnitude between $M^{*}-1$ and $M^{*}+2$ (with $M^{*}=-20.91$, Lin et al. 1996) and with photometric data available in the catalog of $\mathrm{MdC} 01$. Using as a reference for the blue excess fraction $\left(f_{\mathrm{B}}=N_{\mathrm{B}} / N_{\text {tot }}\right)$ the same g-r vs $\mathrm{r}$ color-magnitude relation as in $\mathrm{MdC} 01$, we derived $f_{\mathrm{B}}=0.14 \pm 0.04\left(N_{\text {tot }}=83\right)$ when the photometric members are included and $f_{\mathrm{B}}=0.16 \pm 0.07\left(N_{\text {tot }}=32\right)$ for the sample restricted to spectroscopically confirmed members. Correcting for the color bias discussed in Appendix A, we find that a lower limit to the blue fraction is $f_{\mathrm{B}}=0.14$.

We also defined a new color-magnitude relation (CMR) based on the $B$ and $V$ bands and restricted to morphologically confirmed early-type galaxies. The $B-V$ color index was that originally used in BO84. As in BO84, the blue galaxies were defined as those with a $B-V$ lower by $0.2 \mathrm{mag}$ with respect to the CMR. Using the same luminosity range and spatial domain as before, we found $f_{\mathrm{B}}=0.18 \pm 0.04\left(N_{\text {tot }}=127\right)$ and $0.25 \pm 0.06\left(N_{\text {tot }}=68\right)$ for the photometric and spectroscopic cluster sample respectively $\left(f_{\mathrm{B}}=0.19\right)$ if the latter is corrected for color bias.

Our results arguably favor a rather large blue fraction for this cluster - at least 0.15 - which is free from uncertainties in the background subtraction.

10 With the cosmology adopted by Margoniner et al. (2001), $0.7 \mathrm{Mpc}$ corresponds to $3^{\prime}$, i.e. within our field of view. 


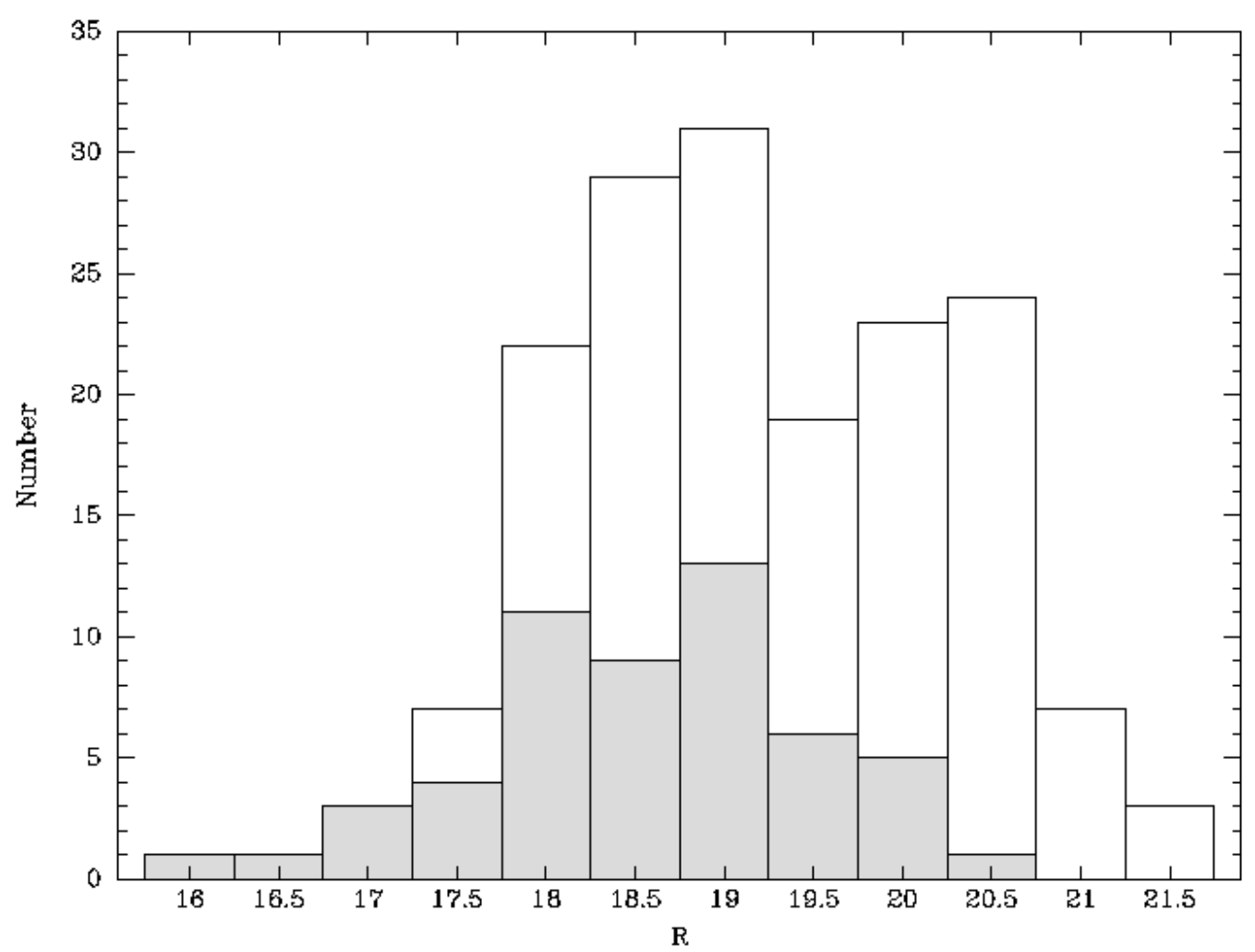

Fig. A.1. $R$-band magnitude histogram of photometrically and spectroscopically (shaded) confirmed cluster members within the area covered by the photometric redshift survey of Dye et al. (2001).

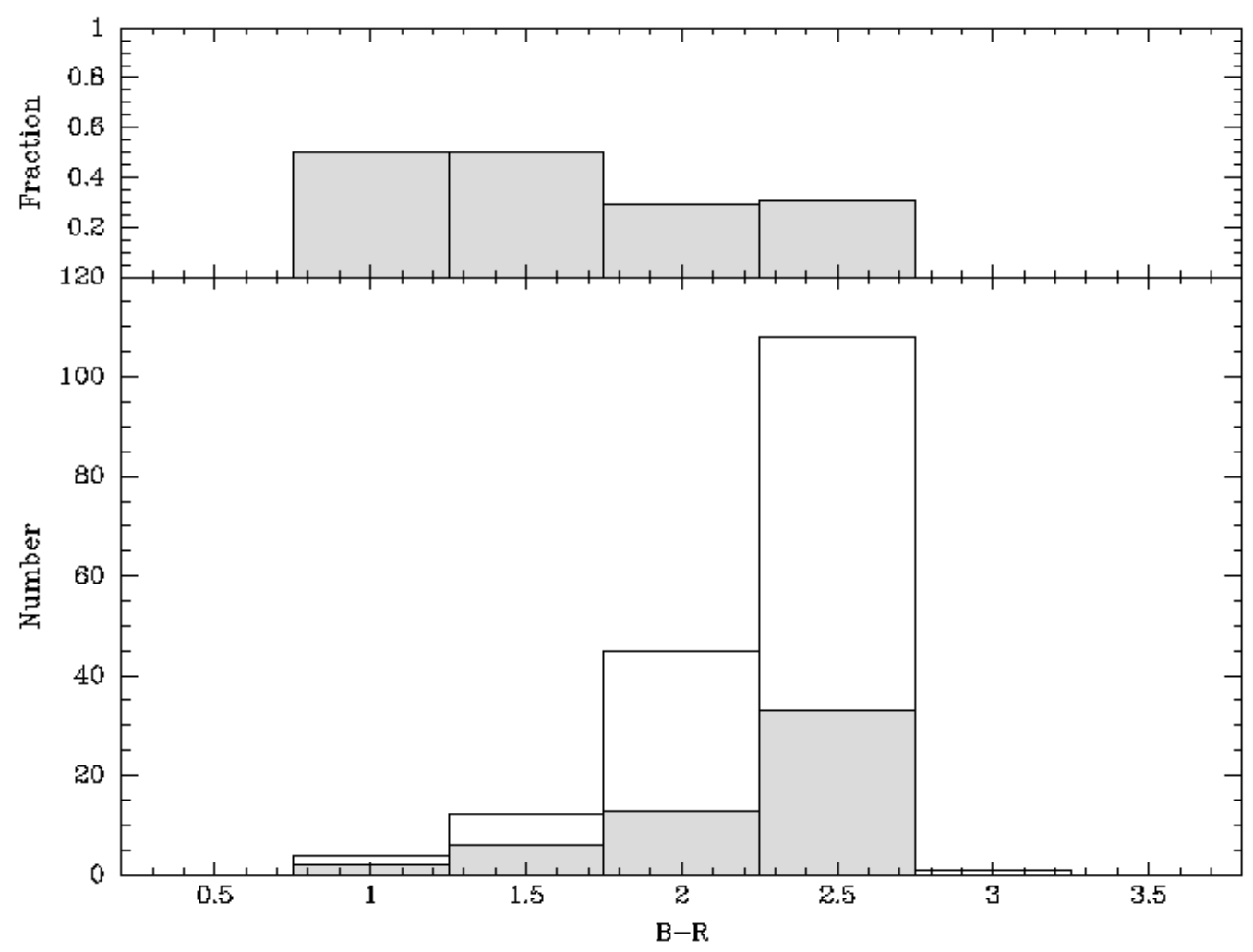

Fig. A.2. $B-R$ color index histogram of photometrically and spectroscopically (shaded) cluster members within the area covered by the photometric redshift survey of Dye et al. (2001). The top panel shows the number ratio of spectroscopically to photometrically confirmed members within each color bin. 


\section{References}

Abraham, R. G., Smecker-Hane, T. A., Hutchings, J. B., et al. 1996, ApJ, 471, 694

Aussel, H., Cesarsky, C. J., Elbaz, D., \& Starck, J. L. 1999, A\&A, 342, 313

Balogh, M. L., \& Morris, S. L. 2000, MNRAS, 318, 703

Balogh, M. L., Morris, S. L., Yee, H. K. C., et al. 1999, ApJ, 527, 54

Balogh, M. L., Navarro, J. F., \& Morris, S. L. 2000, ApJ, 540, 113

Barger, A. J., Aragon-Salamanca, A., Smail, I., et al. 1998, ApJ, 501, 522

Bertin, E., \& Arnouts, S. 1996, A\&AS, 117, 393

Biviano, A., Katgert, P., Mazure, A., et al. 1997, A\&A, 321, 84

Boselli, A., Lequeux, J., Sauvage, M., et al. 1998, A\&A, 335, 53

Butcher, H., \& Oemler, A. 1984, ApJ, 285, 426

Cesarsky, C. J., Abergel, A., Agnese, P., et al. 1996, A\&A, 315, L32

Charlot, S., \& Longhetti, M. 2001, MNRAS, 323, 887

Chary, R., \& Elbaz, D. 2001, ApJ, 556, 562

Couch, W. J., Balogh, M. L., Bower, R. G., et al. 2001, ApJ, 549,820

Couch, W. J., \& Sharples, R. M. 1987, MNRAS, 229, 423

de Propris, R., Stanford, S. A., Eisenhardt, P. R., et al. 1999, AJ, 118, 719

Dressler, A. 1987, in Nearly Normal Galaxies, From the Planck Time to the Present (Springer-Verlag, New York), 276

Dressler, A., Smail, I., Poggianti, B. M., et al. 1999, ApJS, 122, 51

Dye, S., Taylor, A. N., Thommes, E. M., et al. 2001, MNRAS, 321,685

Elbaz, D., Cesarsky, C., Chanial, P., et al. 2001, A\&A, submitted

Fabricant, D. G., McClintock, J. E., \& Bautz, M. W. 1991, ApJ, 381, 33

Fadda, D., Elbaz, D., Duc, P.-A., \& Flores, H. 2000a, in ASP Conf. Ser. 200: Clustering at High Redshift, 96

Fadda, D., Elbaz, D., Duc, P. A., et al. 2000b, A\&A, 361, 827 (Paper I)

Fasano, G., Poggianti, B. M., Couch, W. J., et al. 2000, ApJ, 542,673

Fisher, D., Fabricant, D., Franx, M., \& van Dokkum, P. 1998, ApJ, 498, 195

Flores, H., Hammer, F., Thuan, T. X., et al. 1999, ApJ, 517, 148

Genzel, R., \& Cesarsky, C. J. 2000, ARA\&A, 38, 761

Girardi, M., Fadda, D., Escalera, E., et al. 1997, ApJ, 490, 56

Gudehus, D. H., \& Hegyi, D. J. 1991, AJ, 101, 18

Hunt, L. K., Vanzi, L., \& Thuan, T. X. 2001, A\&A, 377, 66
Jansen, R. A., Franx, M., \& Fabricant, D. 2001, ApJ, 551, 825

Kennicutt, R. C., Jr. 1998, ARA\&A, 36, 189

Kennicutt, R. C., Jr. 1992, ApJ, 388, 310

Kodama, T., \& Bower, R. G. 2001, MNRAS, 321, 18

Laurent, O., Mirabel, I. F., Charmandaris, V., et al. 2000, A\&A, 359, 887

Lin, H., Kirshner, R. P., Shectman, S. A., et al. 1996, ApJ, 464, 60

Madau, P., Ferguson, H. C., Dickinson, M. E., et al. 1996, MNRAS, 283, 1388

Margoniner, V. E., \& de Carvalho, R. R. 2000, AJ, 119, 1562

Margoniner, V. E., de Carvalho, R. R., Gal, R. R., \& Djorgovski, S. G. 2001, ApJ, 548, L143

Metevier, A. J., Romer, A. K., \& Ulmer, M. P. 2000, AJ, 119, 1090

Miralda-Escude, J., \& Babul, A. 1995, ApJ, 449, 18

Molinari, E., Buzzoni, A., \& Chincarini, G. 1996, A\&AS, 119, 391

Monet, D. 1996, in BAAS, 188, 5404

Morrison, G. E. 2000, in American Astronomical Society Meeting, vol. 197, 5704

Moss, C., \& Whittle, M. 2000, MNRAS, 317, 667

Oliver, S., Rowan-Robinson, M., Alexander, D. M., et al. 2000, MNRAS, 316, 749

Pickles, A. J., \& van der Kruit, P. C. 1991, A\&AS, 91, 1

Poggianti, B. M., Bressan, A., \& Franceschini, A. 2001, ApJ, 550, 195

Poggianti, B. M., Smail, I., Dressler, A., et al. 1999, ApJ, 518, 576

Poggianti, B. M., \& Wu, H. 2000, ApJ, 529, 157

Rola, C. S., Terlevich, E., \& Terlevich, R. J. 1997, MNRAS, 289, 419

Roussel, H., Sauvage, M., Vigroux, L., et al. 2001, A\&A, 372, 406

Sanders, D. B., \& Mirabel, I. F. 1996, ARA\&A, 34, 749

Sanders, D. B., Soifer, B. T., Elias, J. H., et al. 1988, ApJ, 325, 74

Serjeant, S. B. G., Eaton, N., Oliver, S. J., et al. 1997, MNRAS, 289, 457

Smail, I., Dressler, A., Couch, W. J., et al. 1997, ApJS, 110, 213

Smail, I., Edge, A. C., Ellis, R. S., \& Blandford, R. D. 1998, MNRAS, 293, 124

Smail, I., Morrison, G., Gray, M. E., et al. 1999, ApJ, 525, 609

Spinoglio, L., Malkan, M. A., Rush, B., et al. 1995, ApJ, 453, 616

Teague, P. F., Carter, D., \& Gray, P. M. 1990, ApJS, 72, 715

Tran, Q. D., Lutz, D., Genzel, R., et al. 2001, ApJ, 552, 527

Tresse, L., \& Maddox, S. J. 1998, ApJ, 495, 691

Tyson, J. A., \& Fischer, P. 1995, ApJ, 446, L55

Veilleux, S., Kim, D.-C., \& Sanders, D. B. 1999, ApJ, 522, 113

Veilleux, S., \& Osterbrock, D. E. 1987, ApJS, 63, 295 